\title{
Occurrence statistics of magnetic impulsive events
}

\author{
T. Moretto ${ }^{1}$, D. G. Sibeck ${ }^{1}$, and J. F. Watermann ${ }^{2}$ \\ ${ }^{1}$ Laboratory for Extraterrestrial Physics, NASA Goddard Space Flight Center, Greenbelt, Maryland, USA \\ ${ }^{2}$ Danish Meteorological Institute, Copenhagen, Denmark
}

Received: 25 April 2003 - Revised: 20 June 2003 - Accepted: 25 June 2003 - Published: 1 January 2004

\begin{abstract}
In this study, we perform a statistical investigation of magnetic impulse events identified in the Greenland magnetometer stations through the years 1995-2001. We focus on occurrence statistics that can be determined reliably with an automatic event identification procedure. Durin the first two years we observed almost $40 \%$ more events than in the following years. Season is not a significant factor in event occurrence. Event occurrence peaks near 12:00 UT, corresponding to approximately 10:00 magnetic local time (MLT) at the west coast of Greenland. More events occur prior to local noon than after. Event days are not distributed evenly. Large amplitude events, particularly, tend to appear on consecutive days. Events are observed at lower latitudes at earlier local times in a way consistent with the projection of the outer magnetospheric boundary into the ionosphere. Event latitude depends on dipole tilt angle in a manner similar to that reported for the cusp. Events occur during intervals of enhanced $K_{p}$. The main reason for this is that the events themselves contribute to the $K_{p}$ index. Events exhibit a preference for high solar wind velocity. In particular, the large amplitude events occur during high-speed streams. A slight preference for lower density and more radial interplanetary magnetic fields, as compared to the nominal solar wind distribution, is also observed. However, both the nominal solar wind and event distribution exhibit large differences from year to year, indicating that events occur under a broad range of conditions.
\end{abstract}

Key words. Ionosphere (ionospheric disturbances) - Magnetospheric physics (magnetosphere-ionosphere interactions; solar wind-magnetosphere interactions)

\section{Introduction}

Transient (5-20 min) variations in the ground magnetic field at high latitudes are very common. On the dayside, where

Correspondence to: T. Moretto

(tmoretto@lepvax.gsfc.nasa.gov) they clearly are not directly associated with substorm processes, they go under the generic name of magnetic impulse events (MIEs). A large portion of MIEs result as the magnetic signature of ionospheric traveling convection vortex (TCV) events as first demonstrated by Friis-Christensen et al. (1988) and Glassmeier et al. (1989). Potentially, MIEs can be caused by any of a long list of processes in the outer magnetosphere. Early suggestions included bursty reconnection at the magnetopause (Glassmeier et al., 1984; Lanzerotti et al., 1986) and magnetopause motion driven by pressure pulses or abrupt changes in the interplanetary magnetic field (IMF) (Friis-Christensen et al., 1988; Sibeck et al., 1989). Currently, evidence seems to be mounting in support of foreshock generated density variations in the solar wind as an important source of TCVs (Sibeck, 1995; Sibeck and Korotova, 1996; Sitar et al., 1998; Sibeck et al., 1999; Zesta et al., 1999; Moretto et al., 2002; Murr et al., 2002; Murr and Hughes, 2003). Particularly, Murr and Hughes (2003) very convincingly demonstrate one-to-one correspondence between TCV events and foreshock cavity-like signatures for 30 out of their 31 events. This ties in well with statistical studies of MIEs that have shown them to occur more frequently prior to noon local time than after and to favor more radial IMF orientation (Glassmeier et al., 1989; Lanzerotti et al., 1991; Konik et al., 1994; Sibeck and Korotova, 1996; Zesta et al., 2002) as also expected for the cavities (Sibeck et al., 2001). Other predictions, however, are not clearly confirmed by existing MIE and TCV statistics, a prominent example being the preference for high solar wind speeds (Sibeck et al., 2001). Furthermore, many discrepancies exist between the earlier statistical studies causing confusion about their results regarding, for example, seasonal and local time occurrence rates (e.g. discussions in Sibeck and Korotova, 1996; Zesta et al., 2002).

One obvious source of confusion is the fact that the definitions of MIE and TCV events are intimately tied to the observational set-up with which they are recorded. For example, it takes a dense network of stations, with substantial latitudinal and longitudinal coverage, unambiguously to 


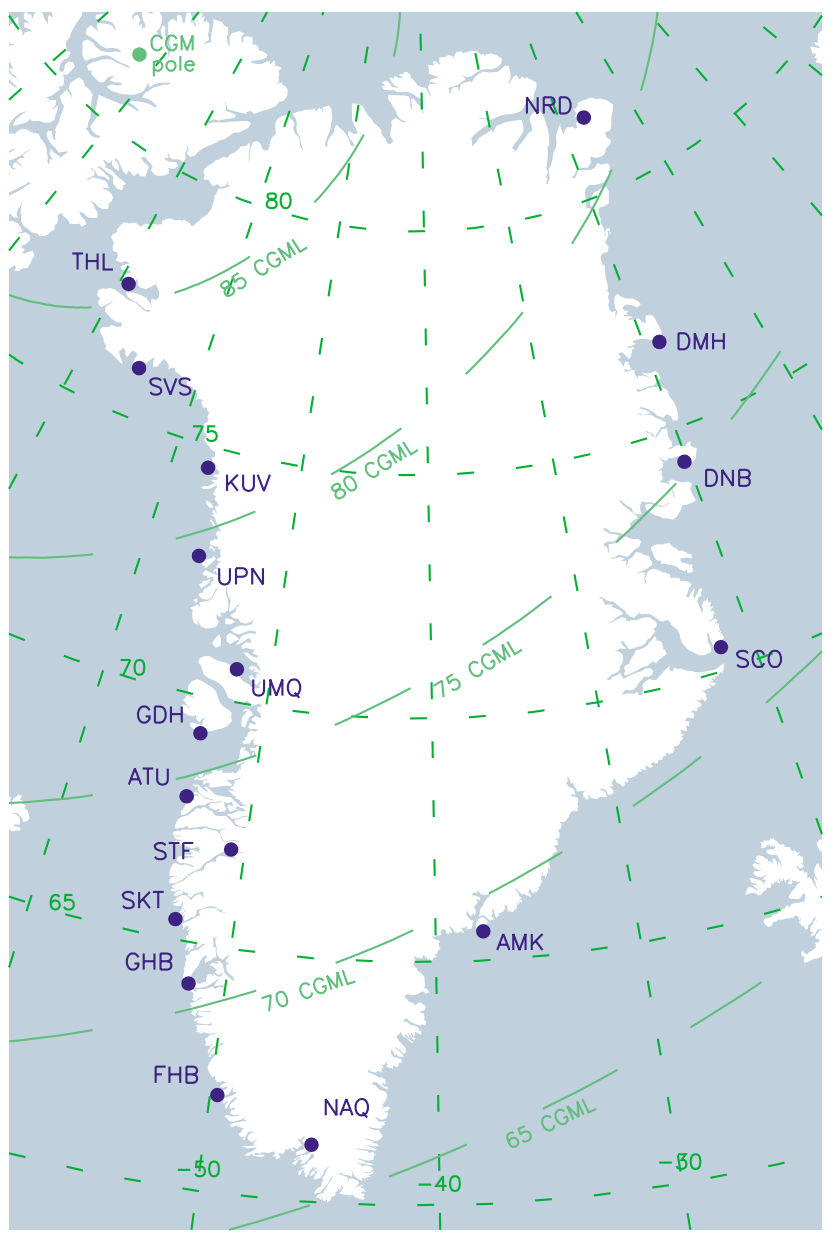

Fig. 1. A map of the Greenland stations used in the study is shown. Stations are named by their three-letter IAGA acronym. Corrected geomagnetic latitude curves as well as the approximate location of the geomagnetic pole are included for reference.

confirm the traveling vortex property of an event. This has been done successfully but, inevitably, only for a limited number of case-studies (Moretto et al., 1997; Zesta et al., 1999; Murr et al., 2002; Amm et al., 2002). Consequently, the exact relationship between MIE and TCV events has not been worked out. On the other hand, the closing of filaments of field-aligned currents from the outer magnetosphere into and out of the ionosphere that drive the transient ionospheric signature inherently produces vortical structures in the ionosphere (see e.g. Southwood and Kivelson, 1989, 1991). This makes the exact verification of the vortical structure of MIEs less important. What is more important is the definition of an appropriate scale size for the signatures under consideration. Inherent in the definition of TCVs has been the fact that the structures must be large and long-lived enough to be identified and followed in their propagation over a large number of magnetometer stations. Notably, TCVs were first verified in the observations from the network of stations in Greenland spanning almost $20^{\circ}$ in latitude and $3 \mathrm{~h}$ of local time in longitude (Friis-Christensen et al., 1988). Statistical studies in the past have dealt with this question in a variety of ways. Studies based on single stations adopted requirements on the amplitude of the events as well as specific waveforms for the magnetometer signature (Glassmeier et al., 1989; Lanzerotti et al., 1991). Sibeck and Korotova (1996) used a widely spread network of stations and required that the signature be observed by at least three stations at a certain amplitude. Similarly, Vorobjev et al. (1999) and Zesta et al. (2002), who both used a small number of stations widely spread in local time, required signatures to be observed at more than one of their stations at a certain amplitude. Zesta et al. (2002), in addition, applied a waveform requirement to further ensure the compatibility of their selected events with the TCV interpretation. Finally, the studies of Moretto and Yahnin (1998), Lühr et al. (1998) and Murr and Hughes (2003) involved only a small number of events making it feasible to manually confirm each of them as proper TCV events.

Based on experience from earlier statistical studies we undertake here a comprehensive statistical analysis of MIE events identified in the Greenland magnetometers over several years from 1995 through 2001. The Greenland network, with its latitudinal and longitudinal coverage, is uniquely suited for this purpose. Particularly, these are the first statistics based on a dense meridional chain of stations. In addition, careful consideration is given to event identification and selection procedures. Our results confirm and solidify many of the previous findings regarding TCVs and bring new, interesting relationships to light. In addition, our study clarifies and settles most of the discrepancies between the existing results.

\section{Method and data}

The study is based on ground based magnetic variation measurements from the network of stations in Greenland operated by the Danish Meteorological Institute (Wilhjelm and Friis-Christensen, 1976). Figure 1 shows a map of the stations identified by their IAGA three-letter names.

The network consists of two near-meridional chains. One chain of 12 stations is spread along the western coast at magnetic local time (MLT) approximately UT $-2 \mathrm{~h}$ and covering corrected geomagnetic (CGM) (Papitashvili and King, 1993; Gustafsson et al., 1992)) latitudes $66^{\circ}-86^{\circ}$. The other consists of 4 stations along the eastern coast at MLT approximately UT $+1 \mathrm{~h}$ and CGM latitudes $72^{\circ}-81^{\circ}$. The station AMK, which often is considered part of the eastern chain, had many problems with erroneous data points (spikes) in the measurements during the period of our study and therefore was excluded from our selection procedure.

\subsection{Event identification algorithm}

In the following, we give a detailed account of how transient events (MIE events) are identified in this study. Our strategy has been to derive a purely automatic event identification algorithm. That is, we wanted to avoid visual inspection of 
magnetograms, as has been the case for nearly all studies in the past (Lanzerotti et al., 1991; Glassmeier et al., 1989; Sibeck and Korotova, 1996; Zesta et al., 2002), as this necessarily introduces subjectivity to the selection procedure. For the same reason, as well as for practical reasons given the large scope of our study, we have not included manual determination of the consistency between the magnetic variations and traveling ionospheric current vortices as, for example, in Moretto and Yahnin (1998). Further, we did not want to limit our search to events that fit a specific current vortex model, e.g. with predefined number and size of vortices as in the study of Clauer and Petrov (2002). On the other hand, we wanted, as far as possible, to make our selection criteria consistent with the magnetic signatures of traveling convection vortices. With these considerations, we adopted the following algorithm for event identification and selection: (1) Only the horizontal components of the magnetic variation vectors are used; these are rotated into components $H 1$ and $H 2$, where latter is directed along the local direction of constant CGM latitude (positive towards East) and the former of which is perpendicular to this (positive towards North). (2) A high-pass filter is applied to the measurements by subtracting a 20-min running mean. (3) For each station, extrema are identified separately in the variation time series of the $H 1$ and $H 2$ components. To avoid problems with instrument noise, the absolute value of the variation at an extremum is required to be larger than $10 \mathrm{nT}$. To avoid problems with occasional spike errors in the measurements, the rate of change of the variation on either side of an extremum is required to be less than $10 \mathrm{nT} / \mathrm{s}$. (4) The range of the variations (range $=$ maximum value - minimum value) in a 20 -min window around an extremum is required to be more than $50 \%$ larger than the range values for the 20-min intervals on either side of this window. Amongst extrema (for the same station and the same component), which satisfy this criterion and occur within 20 min of each other, only the one with largest amplitude variation is selected. (5) To constitute a potential event, extrema fulfilling all the above requirements must occur in the same component at least at two west coast stations within 10 min of each other. If two separate events, identified in this way, occur within $20 \mathrm{~min}$ of each other, it means that neither is truly transient according to our definition and they are both discarded. Finally, for an event to be included in this study, we require that an extremum (also fulfilling all the requirements) is detected within 10 min of the time of the event in the other component at least at one station on the west coast and in both components on the east coast (although not necessarily at the same station).

The last requirement focuses the study on transient events that are most likely to be signatures of traveling convection vortices. Demanding that a clear transient signature is observed in both horizontal components greatly enhances the probability that the magnetic variations will match an interpretation in terms of overhead traveling current vortices (see, for example, Lühr and Blawert, 1994). Similarly, requiring that a clear signal is also observed on the east coast (displaced by approximately $3 \mathrm{~h}$ in local time), greatly enhances the probability that the event is the signature of a coherent structure that propagates over a significant distance (from one coast to the other) (see, for example, Moretto and Yahnin, 1998).

For most of the period covered in the study, measurements are also available from at least one of the stations in the MAGIC-array located in the centre of Greenland. These observations may provide further information on the structure and propagation properties of the events. The latitudinal extent of the array, however, is very limited (about $2^{\circ}$ in CGMlatitude). Consequently, in order not to introduce a latitudinal bias into our results, we have not used measurements from these stations for the event identification.

Identification of events has been limited to the UT-h between 08:00 and 17:00. This ensures that nearly all stations are on the dayside (06:00-18:00 MLT). The real obstacle to expansion of this interval, however, is the interference of substorm current systems, which may be identified as transients by the criterion we have adopted here. This is particularly a problem in the early evening hours, where we have found that the large amplitude events are completely dominated by substorm events for times later than 17:00 UT.

\subsection{Amplitude criterion}

In contrast to most previous studies, we do not explicitly apply an amplitude criterion for the identification of events. Naturally, though, some of the other requirements, for example requiring the amplitude variations of the event to be more than $50 \%$ larger than those immediately before and after, may indirectly have such an effect. The implications for the various event distributions of imposing a direct amplitude criterion has been a topic of discussion in many previous papers (e.g. Sibeck, 1993; Lin et al., 1995; Sibeck and Korotova, 1996; Zesta et al., 2002). To investigate this effect, we define two subgroups of events. In one group we require a maximum amplitude variation (in either component at either the east or west coast part of the event) of more than $100 \mathrm{nT}$. In the other group we require that the overall maximum amplitude variation observed in the event is less than $40 \mathrm{nT}$.

\subsection{Auxiliary data}

To investigate statistical relationships between the MIE events and general geomagnetic activity levels, we use the standard 3-h geomagnetic activity index $K_{p}$. In addition, we analyze the influence of average solar wind conditions on event occurrence by invoking hourly averages of the interplanetary magnetic field (IMF) and solar wind plasma parameters provided in the OMNI database at the NSSDC (National Space Science Data Center). We have found that the strongest effects are seen when the hourly average for the one-hour interval immediately preceding an event is used for comparison. This value is determined from the hourly OMNI data by simple interpolation. 


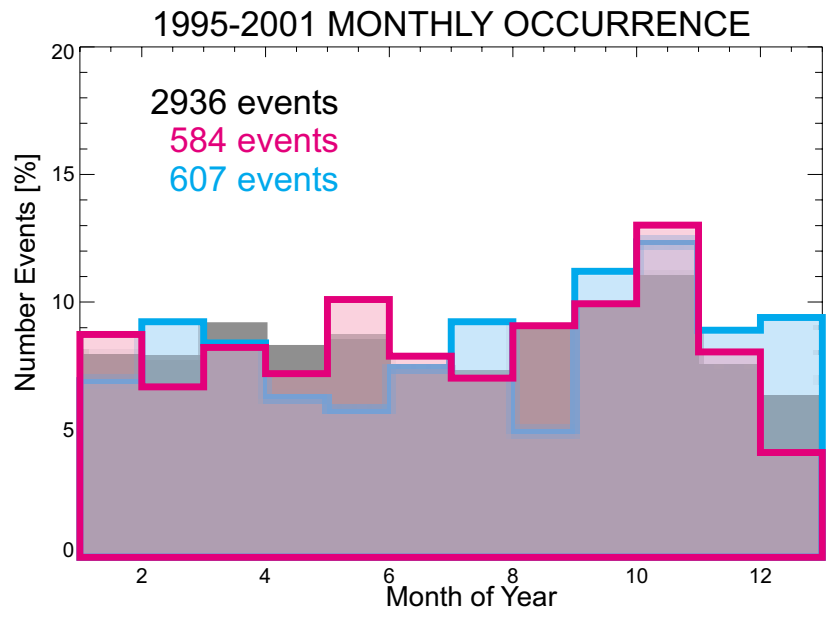

Fig. 2. Relative monthly occurrence for all events observed through 1995-2001 are displayed as the gray block diagram. Overlaid are the monthly occurrence distributions for the subgroups of large and small amplitude events drawn in magenta and cyan colors, respectively. The number of events in each group is written in the corresponding color in the plot. Relative occurrence is displayed as percentage of the total number of events in each case.

\section{Results and discussion}

We have analyzed data for the years 1995 through 2001. The number of events found for all seven years totals 2936 , of which 584 fulfill the large amplitude and 607 fulfill the small amplitude requirements. Table 1 lists the number of events for each year and also gives the numbers for the two subgroups of large amplitude and small amplitude events. The largest number of events in a single year (523) was observed in 1996 and the lowest number was observed in year 2000 (359). We see that the two subgroups each account for roughly $20 \%$ of the total events, although some variations in the division are observed between the individual years.

When comparing these statistics with results from previous studies one must bear in mind differences in station coverage; time-period (year); and event identification criteria, all of which make direct comparisons difficult. The first statistical study was conducted by Glassmeier et al. (1989) and was based on a single station at $68^{\circ}$ magnetic latitude. Events were identified by visual inspection. No direct amplitude criterion was applied but specific wave-forms (unipolar in the east-west component and bipolar in the north-south component) and "clear" events were required. During three years from 1975 to 1979, only 82 events were identified. Lanzerotti et al. (1991) used two nearly conjugate stations at roughly $74^{\circ}$ magnetic latitude. Transient events were identified by an automatic algorithm similar to the one used here but, in addition, events were confirmed by visual inspection. An amplitude criterion of roughly $50 \mathrm{nT}$ was applied. The study covered 17 months from July 1985 to December 1986, during which approximately 240 events were identified at each station. More recently, two comprehensive studies have been completed. Sibeck and Korotova (1996) used a set of high latitude stations widely distributed in latitude and local time; they identified transient events by visual inspection and applied an amplitude criterion of roughly $30 \mathrm{nT}$. A total of 360 events over a period of 11 months in 1986 was registered. Zesta et al. (2002) used three stations at $75^{\circ}-77^{\circ}$ magnetic latitude and separated by roughly $3 \mathrm{~h}$ in local time. Transient events were identified by visual inspection and an amplitude criterion of roughly $50 \mathrm{nT}$ was applied. A total of 443 events was found during one year from mid 1992 to mid 1993. The occurrence rates found in this study are consistent with the numbers reported in these last two studies.

Clauer and Petrov (2002) reported very different occurrence rates. Like the present study, their study utilized the chain of stations at the west coast of Greenland. They applied an automatic event selection algorithm based on vortical current structure identification. They did not apply an amplitude criterion and did not include any criterion to require events to be transient. For the year 1996, they identified 22500 vortices (events). While we cannot claim to completely understand the discrepancy between this result and both those quoted above and ours, we believe that a major reason is the lack of focus on transient events in their study. Another important difference between their selection criteria and ours is that they do not require events to be seen coherently over a range of longitudes. This means that some of their events could be very short-lived or rapidly changing (they require consistent model fits for only 8 consecutive data points, which equals $160 \mathrm{~s}$ ).

In the following sections, we present detailed statistical analyses of the events and their characteristics and discuss our results in terms of previous findings. We shall compare our findings to the results of the earlier studies quoted above, particularly the recent studies of Sibeck and Korotova (1996) and Zesta et al. (2002).

\subsection{Seasonal occurrence}

Figure 2 depicts relative monthly occurrence rates of events for all years combined.

The results for all events together are displayed as the gray block-diagram. This distribution is rather flat but does show a slight tendency for higher occurrence rates during the Autumnal Equinox and smaller occurrence rates during winter solstice. In addition, the distributions for the subsets of large amplitude (magenta line and shading) and small amplitude events (cyan line and shading) are displayed in the figure. These distributions are less smooth due to the lower numbers and resulting less accurate statistics but, overall, are in fair agreement with the full distribution.

Earlier results on this have been rather contradictory and confusing. Glassmeier et al. (1989) reported that their set of TCV events showed a slight decrease in occurrence during the equinoxes, except for a large spike for the month of October. In contrast, Lanzerotti et al. (1991) found that their set of MIE events showed an increase in occurrence during equinoxes. Sibeck and Korotova (1996) found their events to show a minimum in occurrence during summer. Finally, 
Table 1. Number of transient events.

\begin{tabular}{lllllllll}
\hline Year & 1995 & 1996 & 1997 & 1998 & 1999 & 2000 & 2001 & All \\
\hline All events & 516 & 523 & 381 & 387 & 395 & 359 & 375 & 2936 \\
Large amplitude & $126(24 \%)$ & $107(20 \%)$ & $59(15 \%)$ & $71(18 \%)$ & $64(16 \%)$ & $84(23 \%)$ & $73(19 \%)$ & $584(20 \%)$ \\
Small amplitude & $99(19 \%)$ & $105(20 \%)$ & $88(23 \%)$ & $96(25 \%)$ & $72(18 \%)$ & $67(19 \%)$ & $80(21 \%)$ & $607(21 \%)$ \\
\hline
\end{tabular}

Zesta et al. (2002) reported that their events, identified in data covering one year from mid 1992 to mid 1993, show a very slight increase in occurrence during spring equinox and winter solstice. The Glassmeier et al. (1989) study, covering three years, was the only study to cover several years. It is interesting to note that they recorded the largest number of events for the month of October but dismissed this result as possibly spurious. Our distribution also shows the largest number of events during October, particularly for the subgroup of large events which, presumably, resemble most closely the type of events studied by Glassmeier et al. (1989). We note however that this result is not reproduced for all individual years. The monthly distributions for each year of the study are depicted in Fig. 3. Significant fluctuations occur from year to year, rendering the trends observed in Fig. 2 highly questionable. We conclude, that season is only a weak factor, if at all, in the occurrence of the MIE events. This also explains the large discrepancies that exist between the various studies on this point.

\subsection{Local time occurrence}

Figure 4 shows the diurnal distribution of events. The time of an event is defined here as the UT-time at which the largest H2-component variation occurs amongst the west coast stations that observe the event. Given that all signatures of an event are required to occur within a 10-min interval, however, the exact definition has no practical implications for the results shown here. The format of the figure is the same as that of Fig. 2. The approximate UT-times of local magnetic noon at the west coast (14:00 UT) and east coast (11:00 UT) stations, respectively, are indicated in the figure by green triangles. The three distributions in Fig. 4 are very similar. They exhibit a broad peak around 11:00-12:00 UT (corresponding to 09:00-10:00 MLT at the west coast stations), which drops of more sharply towards earlier times than towards later times. The distributions for the large and small amplitude events (cyan and magenta curves, respectively) are both more sharply peaked than the full distribution and the peak of the large amplitude distribution is located slightly earlier.

The UT, or rather corresponding local time, distribution of MIE and TCV events has been the subject of intense discussion (Glassmeier et al., 1989; Lanzerotti et al., 1991; Lin et al., 1995; Sibeck et al., 1996; Sibeck and Korotova, 1996; Vorobjev et al., 1999; Zesta et al., 2002). The distribution we find here is less sharply peaked than those reported in the ear- lier studies. Qualitatively, however, finding a peak in occurrence for pre-noon local times is in agreement with previous findings. The location of the peak also is in reasonable agreement with earlier results, which report peaks around 08:00 10:00 MLT. There is no clear indication of a mid-noon gap (or a separate secondary peak after noon local time) as reported by Lanzerotti et al. (1991) and Sibeck and Korotova (1996) in any of the distributions. Although the incorporation of both east and west coast stations means that we cannot rigorously interpret our results in terms of local time, they are clearly consistent with more events being observed prior to local noon than after, as reported by all previous studies.

Figure 5 displays the diurnal distributions for each year individually. Though rather large variations in the distributions are observed from one year to the next, they all are in qualitative agreement with the results quoted above. They all exhibit a peak in the distribution prior to 13:00 UT; they all exhibit more events prior to local noon than after; only few distributions show indications of a secondary peak after local noon. The substantial variability that is observed for these distributions as well as for the monthly distributions of Fig. 3 indicates that some controlling factors in the occurrence of the MIE events vary significantly from year to year.

Foremost, the exact forms of the occurrence distributions are determined by the selection criteria on which the event identification is based. We have investigated this by imposing a further restriction on our events, namely that they attain an amplitude of at least $30 \mathrm{nT}$ at the station SKT (at roughly $72^{\circ}$ CGM latitude on the west coast). Figure 6 displays the resulting monthly and diurnal distributions. The diurnal distribution now exhibits a better-defined peak prior to local noon and a secondary afternoon peak while the corresponding monthly occurrence distribution now shows a prominent dip in occurrence during the summer months. Both of these features were also found in the study of Sibeck and Korotova (1996). We shall return to these results later when we present the latitude dependencies of our events.

\subsection{Dipole tilt angle occurrence}

To complement the distributions shown in Fig. 2 and Fig. 4 we display, in Fig. 7, the event occurrence as a function of dipole tilt angle. Dipole tilt angle at the time of an event is calculated as the angle between the Earth magnetic dipole axis, as given by the IGRF for the appropriate epoch, and the GSM $Z$-axis, positive towards the Sun (Summer) and negative away from the Sun (winter). These distributions confirm 


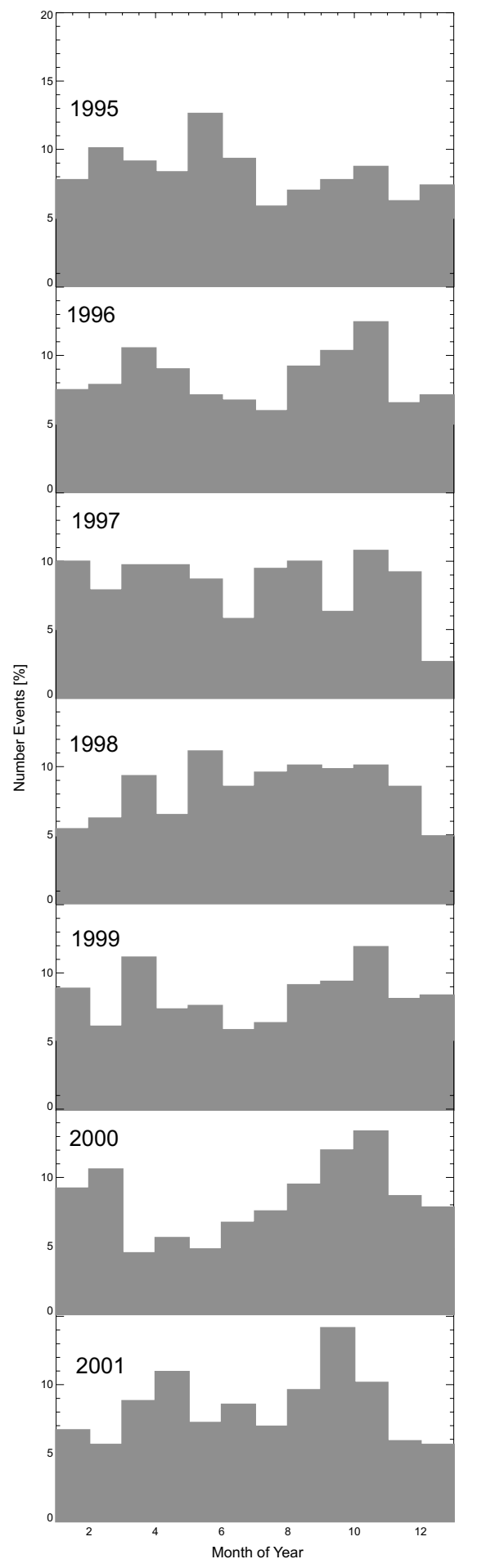

Fig. 3. Relative monthly occurrences for each year are displayed in the panel. The format of the gray block-diagrams is the same as for Fig. 2

the results of Fig. 2 and Fig. 4 and are included here mainly to demonstrate the dipole tilt-angle coverage, which will be used for reference in the discussion, later, of dipole tilt-angle dependency on event parameters.

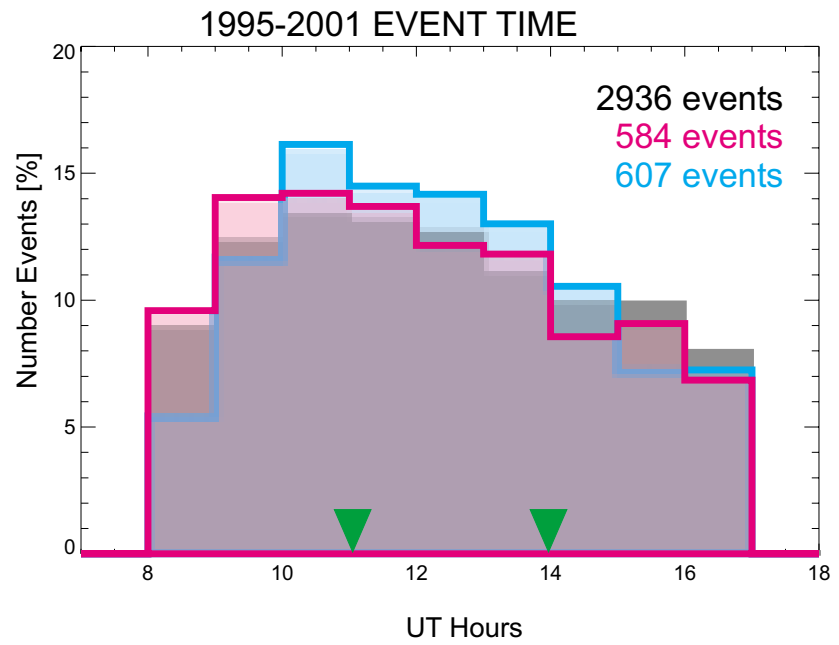

Fig. 4. Relative occurrences as functions of universal time of events. Hourly bins have been used for the UT time. The format of the plot is the same as for Fig. 2. Green triangles mark the approximate UT time of local magnetic noon at the west coast (roughly 14:00 UT) and the east coast (roughly 11:00 UT) of Greenland, respectively.

\subsection{Event separation}

Next, we present a few results on the distribution of events. Figure 8 shows the distribution of the number of events per day. Observing 2936 events over a period of 2555 days (the full seven years of our study) yields an average of 1.2 events per day. In the same way, we observe an average of 0.2 per day for both large and small events. For comparison, we display, in Fig. 8, the values for the Poisson distribution, which represents the expected distribution for randomly distributed events, for these two averages (black and green diamonds, respectively). In all cases, the event distributions have slightly more days with zero events and less days with one event than the random distributions and, correspondingly, more days with three and four events. The only earlier study that displays such a distribution for the events is the one by Sibeck and Korotova (1996). Their distribution most closely resembles the one for the large events and clearly does not match the one found here for all events.

The separation between days on which events are observed gives a different picture, however. Figure 9 shows the distribution of days elapsed between a day observing one or more events and the last such day a value of one means consecutive days. The 2936 events occur on 1637 different days, corresponding to an average separation of 1.56 days between event-days. For the large events, the numbers are 584 events on 440 days giving an average separation of 5.8 days (small events have 607 events on 520 days giving practically the same average). Again, we show for comparison the relevant Poisson distributions, representing the expected distributions for randomly occurring event-days (black and green stars, respectively). The large and small event-days clearly occur more in groups than is consistent with a random distribution of days. The full event distribution exhibits a sim- 


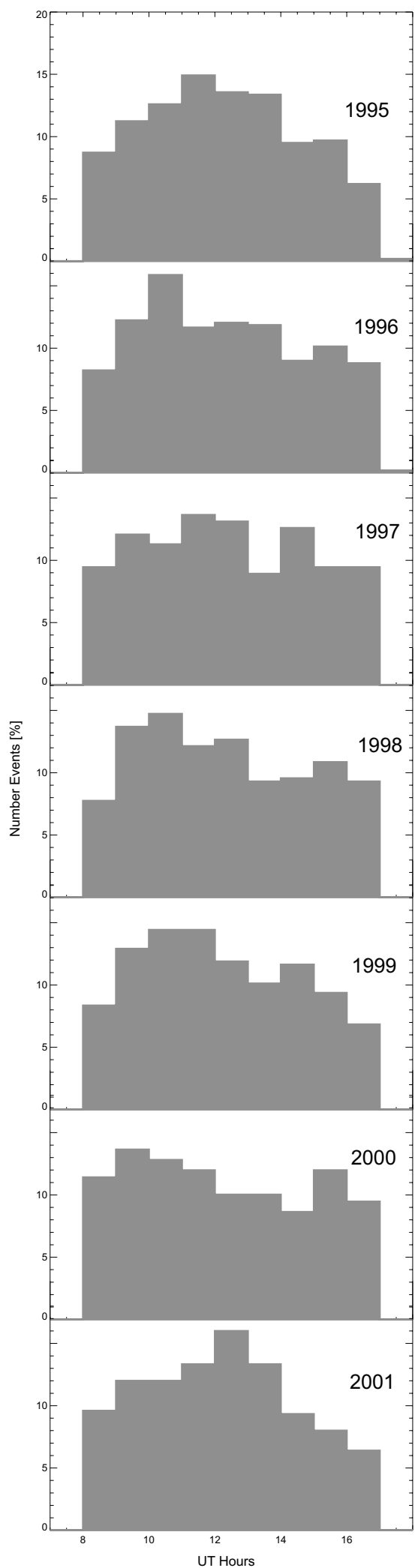

Fig. 5. Relative occurrences, as functions of the universal time of events, displayed individually for each year as shown in the panels. The format of the gray block-diagrams is the same as for Fig. 4 .
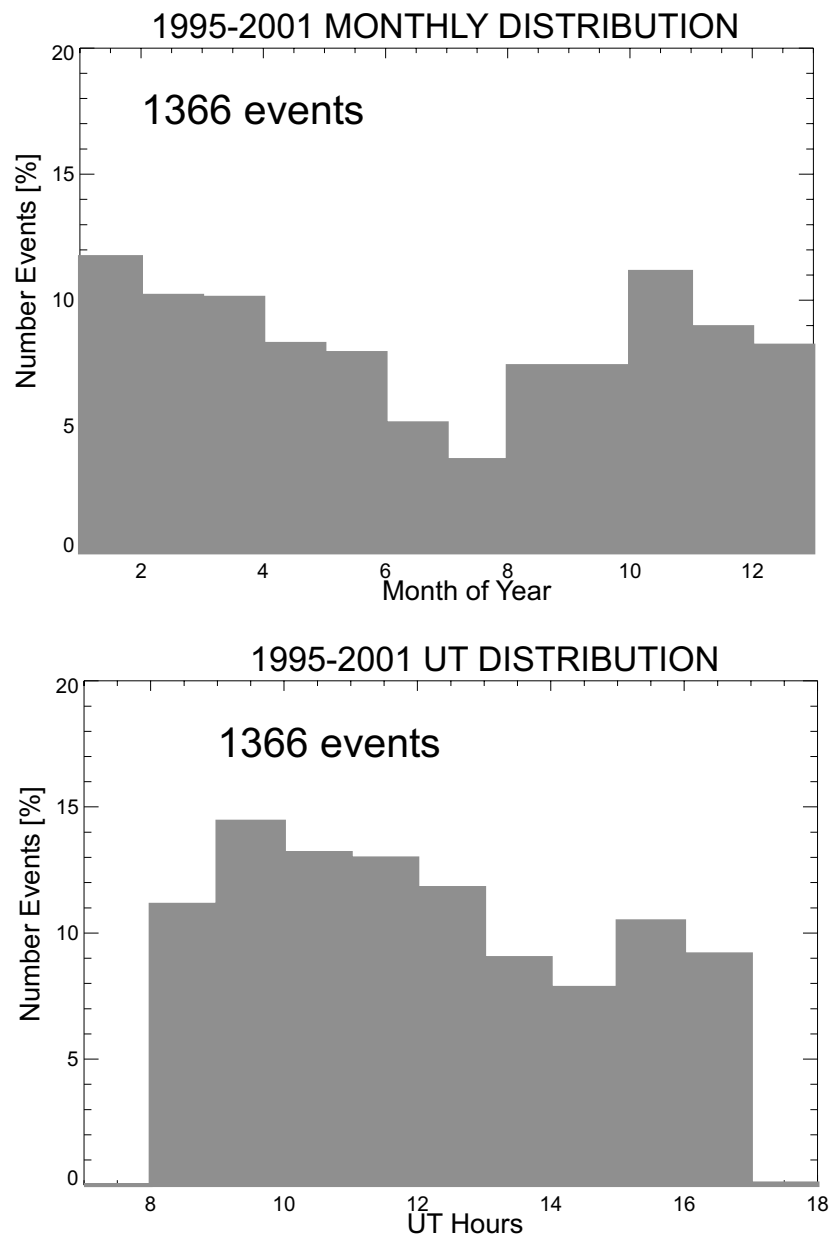

Fig. 6. The upper panel displays the monthly occurrence rates and the lower panel the diurnal distribution of the subgroup of events observed with an amplitude of at least $30 \mathrm{nT}$ at the station SKT. The number of events is shown in each panel.

ilar tendency. Lühr et al. (1998) investigated 19 large amplitude TCV events, which were identified in a set of magnetometers in Northern Scandinavia $\left(66^{\circ}-76^{\circ}\right.$ magnetic latitude) during six months from October 1993 to March 1994. They also found that the event-days were not randomly distributed. More than $40 \%$ of their event-days fall within 5 days of another event-day, which is similar to the numbers we find here for the largest events. However, they observed a second peak in the distribution at 10-15 days, which we do not observe.

\subsection{Amplitude distributions}

Figure 10 displays the distribution of our event amplitudes. Amplitude is taken to be the largest amplitude variation observed amongst all identified extrema (i.e. fulfilling the requirements in our selection criteria) of the event. The distribution peaks in the range from 25 to $75 \mathrm{nT}$, near the lower limit of observable amplitudes, and falls off steadily towards larger amplitudes. Given the differences in amplitude criteria, the distribution seems consistent with those obtained by 


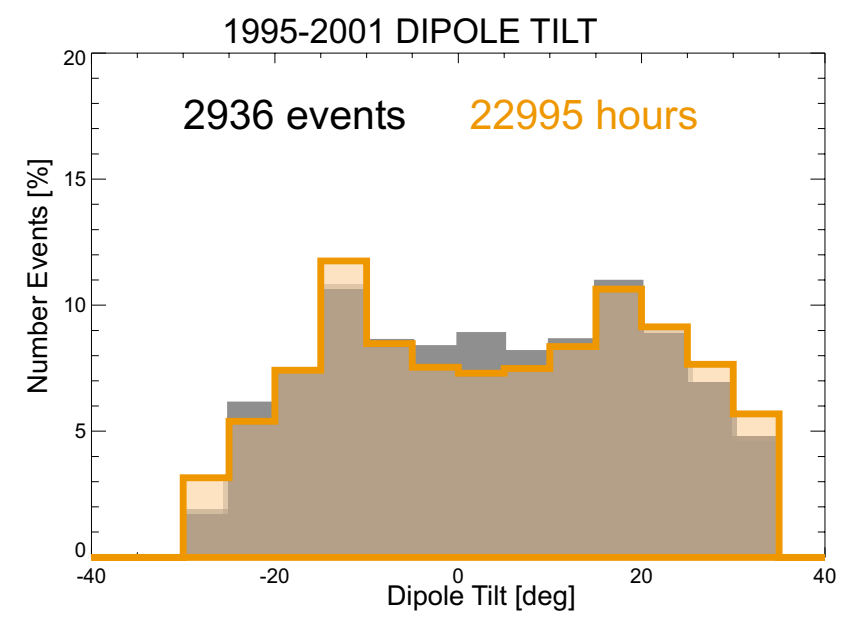

Fig. 7. Relative occurrences shown as functions of dipole tilt angle at the time of events. The format of the plot is largely the same as for Fig. 2. For reference, the background distribution of hourly dipole tilt angle values for the entire period covered in the analysis $(25550 \mathrm{~h})$ is displayed in orange.

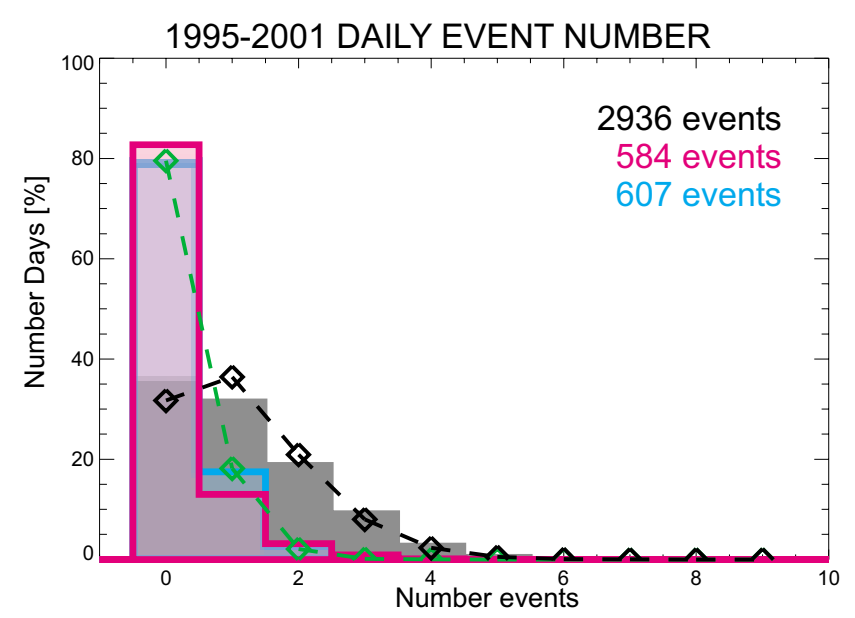

Fig. 8. Distributions of number of events per day are shown for all events (gray block diagram) as well as for the large and small amplitude subgroups (magenta and cyan colors, respectively). Overlaid are the Poisson distributions for averages of 1.2 events per day (black line and diamonds) and 0.2 events per day (green line and diamonds).

Sibeck and Korotova (1996) and Zesta et al. (2002); not surprisingly, it resembles most closely the former, which used the smaller amplitude criterion of the two (roughly $30 \mathrm{nT}$ as compared to roughly $50 \mathrm{nT}$ ).

It has been argued that the observed local time occurrence distribution (peaking in the mid-morning local time hours) might partly be explained by a similar distribution in event amplitudes (Sibeck, 1990; Sibeck and Korotova, 1996). This suggestion is further supported by the observation in several studies (Lühr and Blawert, 1994; Moretto et al., 1997; Zesta et al., 1999; Murr et al., 2002) that individual TCV events first grow in strength and later decay as they travel tailward

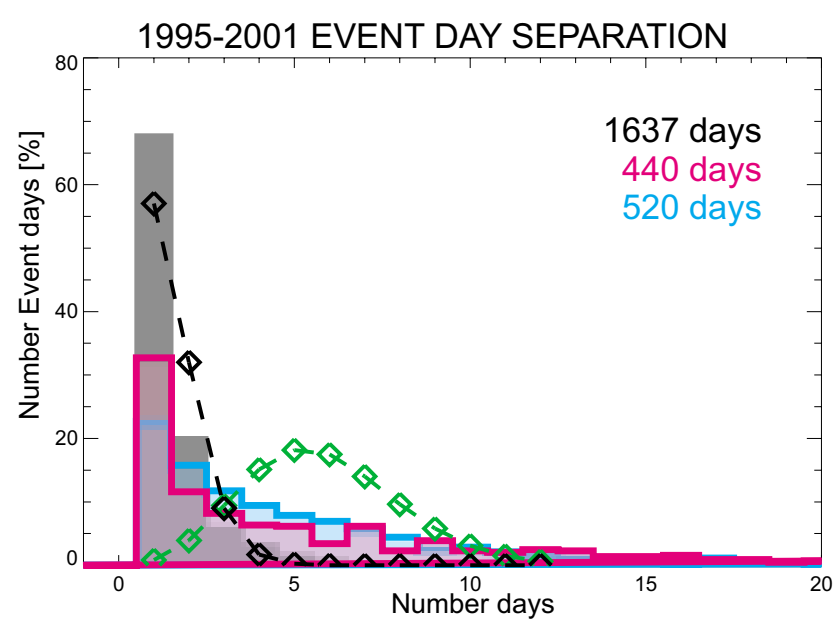

Fig. 9. Distributions of separation between event days shown for all events (gray block diagram) as well as for the large and small amplitude subgroups (magenta and cyan colors, respectively). Overlaid are the Poisson distributions for averages of 1.56 days (black line and diamonds) and 5.8 days (green line and diamonds).

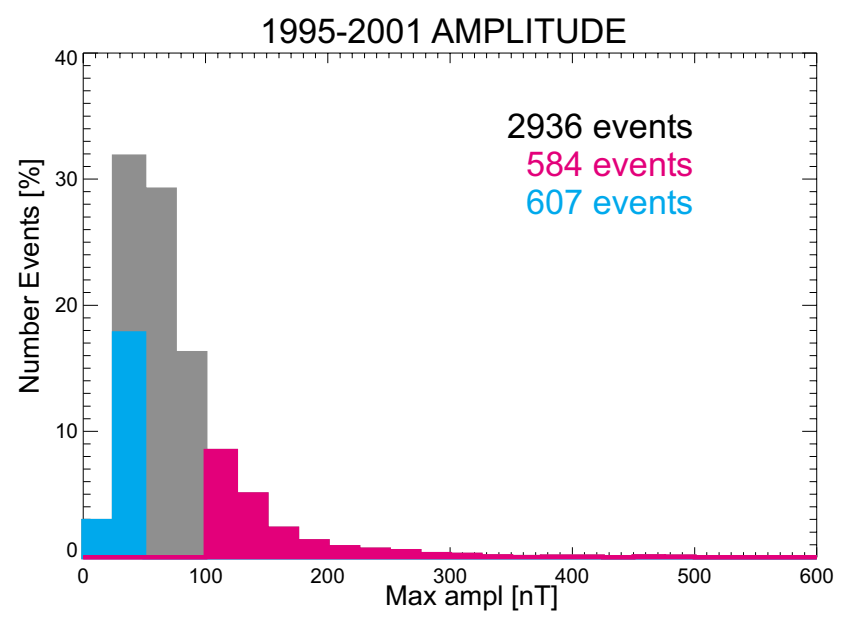

Fig. 10. The distribution of event amplitudes is displayed using bins of $25 \mathrm{nT}$. The large amplitude subgroup of events is marked in magenta and the small amplitude subgroup in cyan.

from their origin near local noon towards dawn. The left panel of Fig. 11 displays the mean amplitude for each onehour bin of event time. Black crosses and error-bars (standard deviation) are for the full set of events, magenta crosses are for the large-amplitude subgroup, and cyan crosses are for the small-amplitude subgroup. All of the curves are flat, which means that the relation between amplitude and local time that has been observed for individual events cannot be confirmed statistically. A similar result is found for the amplitude as a function of dipole tilt-angle shown in the middle panel of Fig. 11. Except for the sharp decrease in mean amplitude observed for the $0-5$ degrees bin, for which we have no explanation at this time, all curves are flat. Specifically, we do not find systematically larger amplitudes for events during summer than during winter which implies that event 

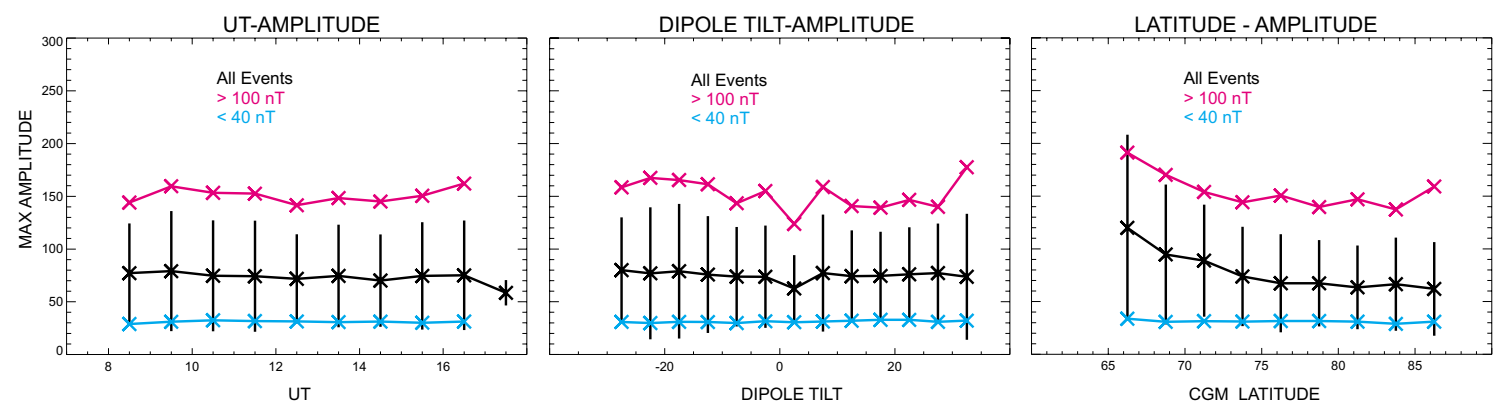

Fig. 11. Mean amplitude of events are shown as functions of UT-time of events (left panel), dipole tilt-angle (middle panel), and event latitude (right panel). In all panels, black crosses and error-bars (standard deviation) present the results for the full set of events; magenta crosses are for the subgroup of large amplitude events, and cyan crosses are for the small amplitude events.

amplitude does not depend strongly on ionospheric conductivity. This agrees with results found in conjugate studies of MIEs and TCVs (Lanzerotti et al., 1991; Kataoka et al., 2001; Murr et al., 2002). The last panel of Fig. 11, in contrast, demonstrates a clear tendency for the amplitude of events to increase with decreasing latitude. This will be explored further in the next section which is devoted to the examination of the latitude dependency of events.

\subsection{Latitude distributions}

Utilizing the full meridional chain in the statistics allows us to analyze the dependencies on event latitude. Latitude of an event is determined as the latitude of the station on the West Coast where the largest amplitude variation in the $\mathrm{H} 2$ component is measured. This corresponds to the latitude where the strongest North-South flow is observed. For an ideal vortex event, this would occur between the two main vortices at the latitude of the center of the vortices.

Figure 12 displays the latitude distribution for all events identified from 1995 through 2001 (gray block-diagram). Also displayed are the distributions for the largest amplitude events (magenta curve and shading) and smallest amplitude events (cyan curve and shading), respectively. Latitude bins of 2.5 degrees have been used to calculate the distribution. Also indicated in the plot is the latitude distribution of the West Coast stations included in the study. The latitude distributions for all three groups of events are mainly located between $70^{\circ}$ and $80^{\circ}$ and peak quite strongly at $73^{\circ}-75^{\circ}$. In addition, the larger amplitude events are shifted slightly towards lower latitudes whereas the smaller amplitude events are shifted towards higher latitudes as compared to the total distribution. In all cases, the distributions fall off rather sharply at low latitudes, around $70^{\circ}$, and fall off more gradually towards high latitudes.

The latitude interval from $70^{\circ}$ to $77^{\circ}$ covers 3 bins, each of which includes two stations, whereas the latitude bins at lower and higher latitudes only cover one station each. The one station bins are more sensitive to occasional lack of data from a single station. Given the very long time-period covered in this study, however, we do not believe this to influence the results significantly. Another issue is the fact that

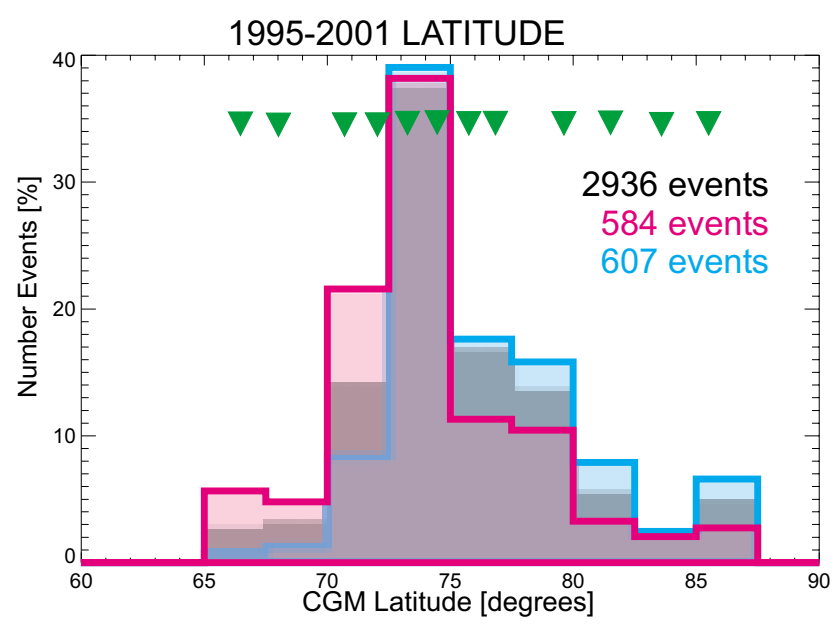

Fig. 12. The distribution of latitudes of events is displayed using bins of 2.5 degrees. The gray block-diagram presents the full set of events, the magenta line and shading the large amplitude subgroup, and the cyan line and shading the small amplitude subgroup. Green triangles mark the location of the Greenland west coast stations.

we require the event to be observed by at least two stations on the West coast. This criterion might seem to favor detection of events within the more densely covered interval from $70^{\circ}$ to $77^{\circ}$; however, we also require events to be registered clearly at the East coast stations. Case-studies of events that remain coherent over at least $3 \mathrm{~h}$ of local time have shown that the latitudinal extent of such events is much larger than a few degrees (e.g. Moretto et al., 1997; Zesta et al., 1999; Amm et al., 2002; Murr et al., 2002). Consequently, we do not expect this small unevenness in latitudinal coverage to significantly influence the latitudinal occurrence statistics as presented. The distribution itself lends further support to this argument. At the lower end, a gap of $2.7^{\circ}$ in latitude exists between $\mathrm{GHB}$ at $70.6^{\circ}$ and $\mathrm{FHB}$ at $67.9^{\circ}$ (all latitudes are for the 1998 epoch), while, at the other end, an almost identical gap of $2.6^{\circ}$ exists between UMQ at $76.9^{\circ}$ and UPN at $79.5^{\circ}$. The distribution, however, only exhibits a sharp increase at the lower gap not a correspondingly sharp decrease at the upper gap. Consequently, we believe both of these features 
in the distribution to be real.

Figure 13 displays the mean latitude as a function of event time and dipole tilt angle. The top two panels show the results for all events (black crosses and error-bars) as well as for the large-amplitude and small-amplitude subgroups (magenta and cyan crosses, respectively). A clear decrease in mean latitude is observed from $77^{\circ}$ at $14: 00$ UT to $74^{\circ}$ at 09:00 UT. The large amplitude events systematically occur at lower latitudes $\left(\right.$ by $1^{\circ}-1.5^{\circ}$ ) and the small amplitude events at higher latitudes (by $0.5^{\circ}-1^{\circ}$ ). This is consistent with the differences in latitude distributions observed in Fig. 12. An increase in event latitude for positive dipole tilt-angles from $75^{\circ}$ at $0^{\circ}$ dipole tilt to $78^{\circ}$ at $25^{\circ}$ is also observed. Since dipole tilt-angle is a function of UT-time, this is not a completely independent result. To estimate the mutual influence of the two effects on each other we display, in the bottom panels of Fig. 13, some additional mean latitude distributions. In the middle left panel we display the UT dependence for events, which occur at times of positive (orange) and negative (green) dipole tilt-angle, respectively. While both curves show the same trend as the full set of events, hence verifying the UT-dependency, the summer events (positive dipole tilt-angle) show an enhanced effect and the winter events a reduced effect. Similarly, the middle right panel of Fig. 13 displays the dipole-tilt dependency separately for events that occur between 09:00 and 11:00 UT (green) and between 13:00 and 15:00 UT, respectively. The subgroup of late events (orange curve) verifies the trend for the full set of events and hence confirms that the observed dipole tilt-angle dependency is not only a result of the UT-dependency.

The latitude of events has previously been mainly considered in event studies, for which extensive data coverage could be gathered and analyzed thoroughly (Lühr et al., 1996; Moretto et al., 1997; Zesta et al., 1999; Amm et al., 2002; Murr et al., 2002, and many others). The movement to lower latitudes as the event propagates away from local noon has been observed for individual events in several cases (Moretto et al., 1997; Murr et al., 2002). Our results here confirm this feature statistically. A few smaller statistical studies have also included results on the latitude distribution of events. Moretto and Yahnin (1998) investigated thirteen TCV events during quiet conditions identified in the Greenland chain observations. They determined latitudes for these events between $70^{\circ}$ and $74^{\circ}$. All these events were in the morning sector (09:00-11:00 MLT) and so the agreement with our findings here is very good. In the statistical study of Sibeck and Korotova (1996), a widely distributed set of stations were used, covering magnetic latitudes between $64^{\circ}$ and $84^{\circ}$; it was found that the largest amplitude signal was most often observed at the station at $74.6^{\circ}$. This, however, was the only station in the study located between $70^{\circ}$ and $76^{\circ}$ so they could not investigate detailed latitude dependencies further. Our results here confirm and solidify this finding. The observed latitude behavior of events explains the large differences in local time occurrence statistics between the earlier studies. If the identification of events is done over a narrow range of latitudes, e.g. by using only a single station, the resulting local time distribution will depend strongly on the latitude of this station. Latitudes lower than roughly $75^{\circ}$ will yield a sharp peak in the early morning together with a secondary peak just after noon (examples are the results of Lanzerotti et al., 1991; Sibeck and Korotova, 1996) while higher latitudes will result in a single broader peak later in the morning (examples are the results of Vorobjev et al., 1999; Zesta et al., 2002). Significant amplitude requirements will further sharpen these trends (an example is the distribution found by Glassmeier et al., 1989).

The bottom left panel of Fig. 13 displays the mean event latitudes (black crosses and error-bars) together with the poleward and equatorward boundaries (green lines) of the statistical Feldstein auroral oval (Feldstein, 1963; Holzworth and Meng, 1975) for quiet conditions $(Q=0)$. It is seen that the local time dependency of the event latitudes closely resembles that of the poleward auroral boundary. Similar local time behavior for the statistical locations of plasma sheet and boundary layer type precipitation regions, which are closely related to the poleward boundary of diffuse aurora, has also been reported from low altitude satellite measurements (Newell and Meng, 1992). This result lends strong statistical evidence in support of the suggestion put forward in several event studies (Lühr et al., 1996; Yahnin et al., 1997; Moretto and Yahnin, 1998; Moretto et al., 2002; Murr et al., 2002) that the ionospheric MIE signature is coupled to a boundary in the outer magnetosphere.

The effect of dipole tilt angle on the statistical location of cusp and boundary layer type precipitation regions from low altitude satellite measurements was reported by Newell and Meng (1989). Their best linear fits for the equatorward boundaries of the cusp and Low-Latitude Boundary Layer (LLBL) are displayed in the bottom right panel of Fig. 13 (upper and lower green curves, respectively) together with the mean latitudes for the full set of events (black crosses and error-bars). The direction of the dipole tilt-angle effect for the events as well as its overall range are similar to the one observed for the cusp but the asymmetric behavior for positive and negative tilt-angle events clearly distinguishes it from both of the precipitation results.

\subsection{Relation to geomagnetic activity}

The top left panel of Fig. 14 displays the distribution of $K_{p}$ values for the events together with the average distribution for the full seven year period covered in the study. In the top right panel the separate distributions for the small and large amplitude subgroups are displayed on top of the full distribution. The events overall exhibit a tendency to occur for slightly elevated $K_{p}$ values. The overall average for the events is $2^{+}$as compared to $2^{0}$ for the general distribution. Both the distribution and the average are in agreement with those reported by Zesta et al. (2002).

The geomagnetic activity level clearly separates the small and large amplitude subgroups. The average is $2^{-}$for small amplitude events and $3^{+}$for large amplitude events. During active periods, only the largest amplitude events stand out 

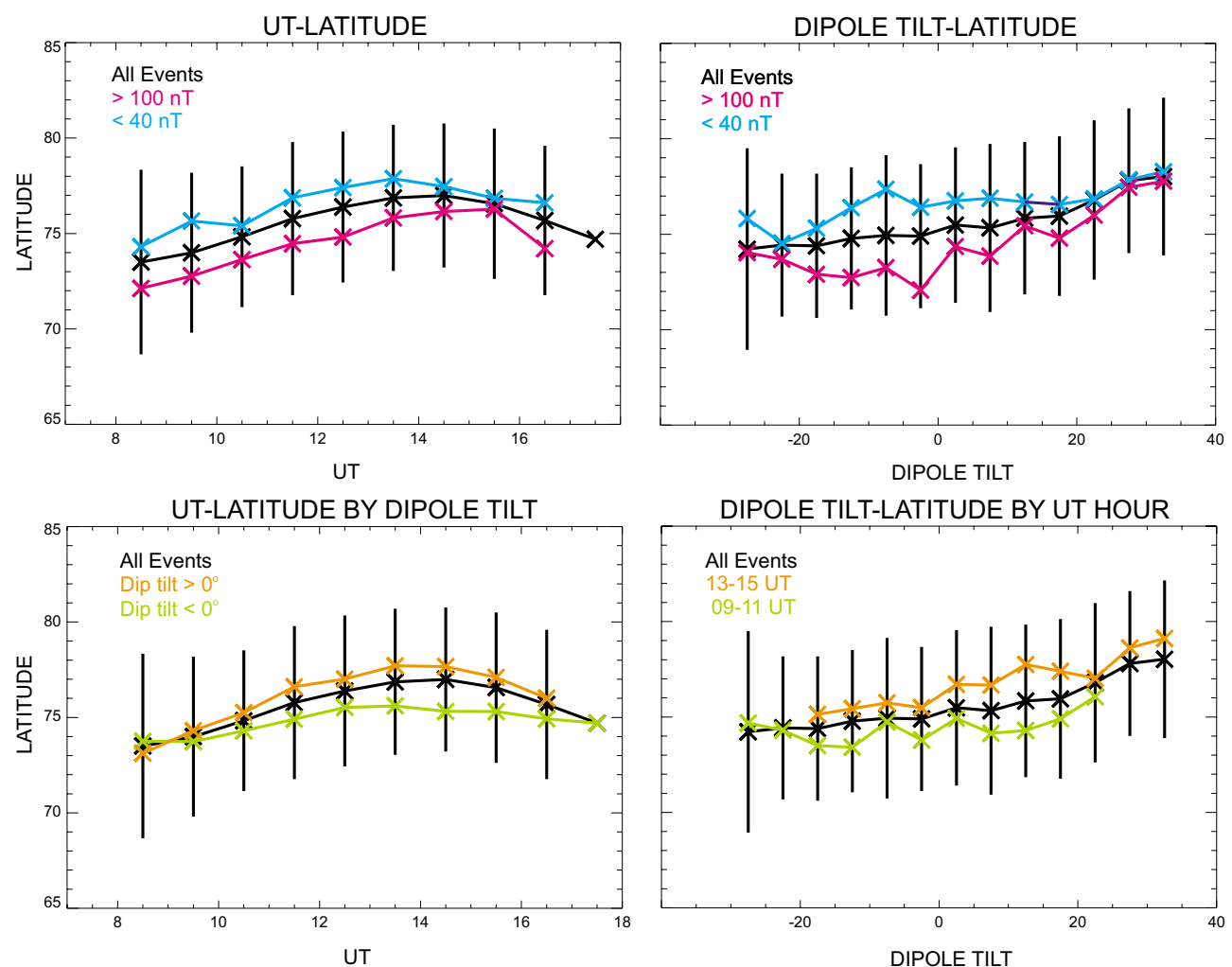

DIPOLE TILT-LATITUDE BY UT HOUR
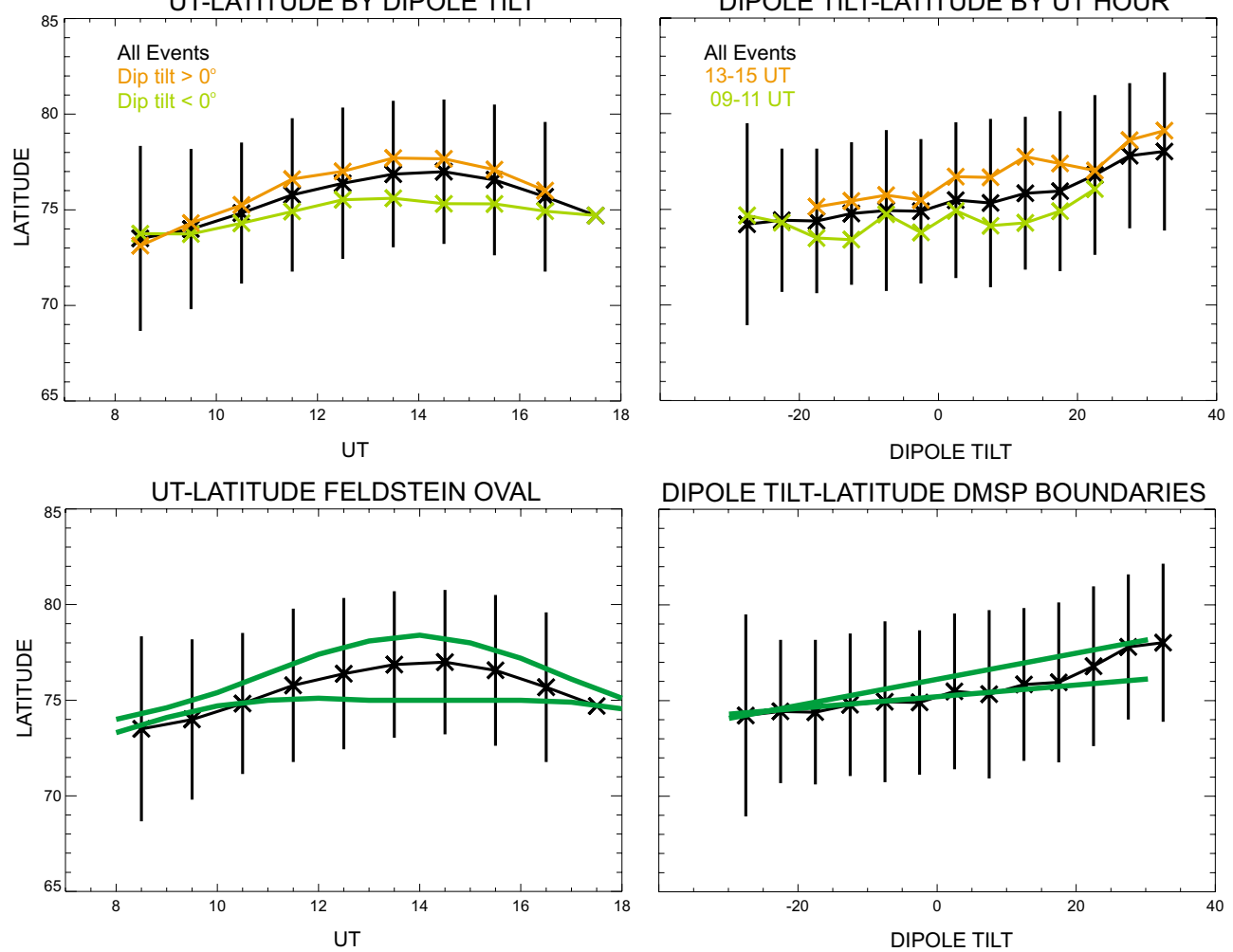

Fig. 13. Mean latitude of events are shown as functions of UT-time of events (left panels) and dipole tilt-angle (right panels). In all panels, black crosses and error-bars (standard deviation) present the results for the full set of events. In the top two panels, magenta crosses are for the subgroup of large amplitude events and cyan crosses are for the small amplitude events. In the middle left panel, orange crosses present the results for the 1776 events that have positive dipole tilt-angle and the green crosses present the 1260 events with negative dipole tilt-angle. In the middle right panel, orange crosses present the results for 620 events occurring between 13:00 and 15:00 UT while the green crosses are for the 762 events that occur between 09:00 and 11:00 UT. In the bottom left panel, green curves mark the location of poleward and equatorward boundaries of the statistical Feldstein oval for $Q=0$ and MLT $=\mathrm{UT}-2$. In the bottom right panel, green curves mark the statistical location of the equatorward boundaries of the Cusp and LLBL for 11:00-13:00 MLT as determined from DMSP particle precipitation data.

and are identified according to our selection criteria. Alternatively, large amplitude events occurring on an otherwise quiet background might themselves be the cause of a registered increase in activity level. As it turns out, the large amplitude events are almost equally likely to occur for southward as for northward IMF (see Fig. 17). In the middle panels of Fig. 14 we display the distributions for these two cases (left and right panel, respectively). The large amplitude events also are almost equally likely to occur during times of solar wind speeds larger and smaller than 500 km/s (see Fig. 15). The distributions for these two cases are displayed in the bottom two panels of Fig. 14. It is seen that high $K_{p}$ values are almost equally likely for large amplitude events during northward as during southward IMF and that, contrary to expectations, more high $K_{p}$ values are associated with the lower than with the higher solar wind speed. We conclude from 

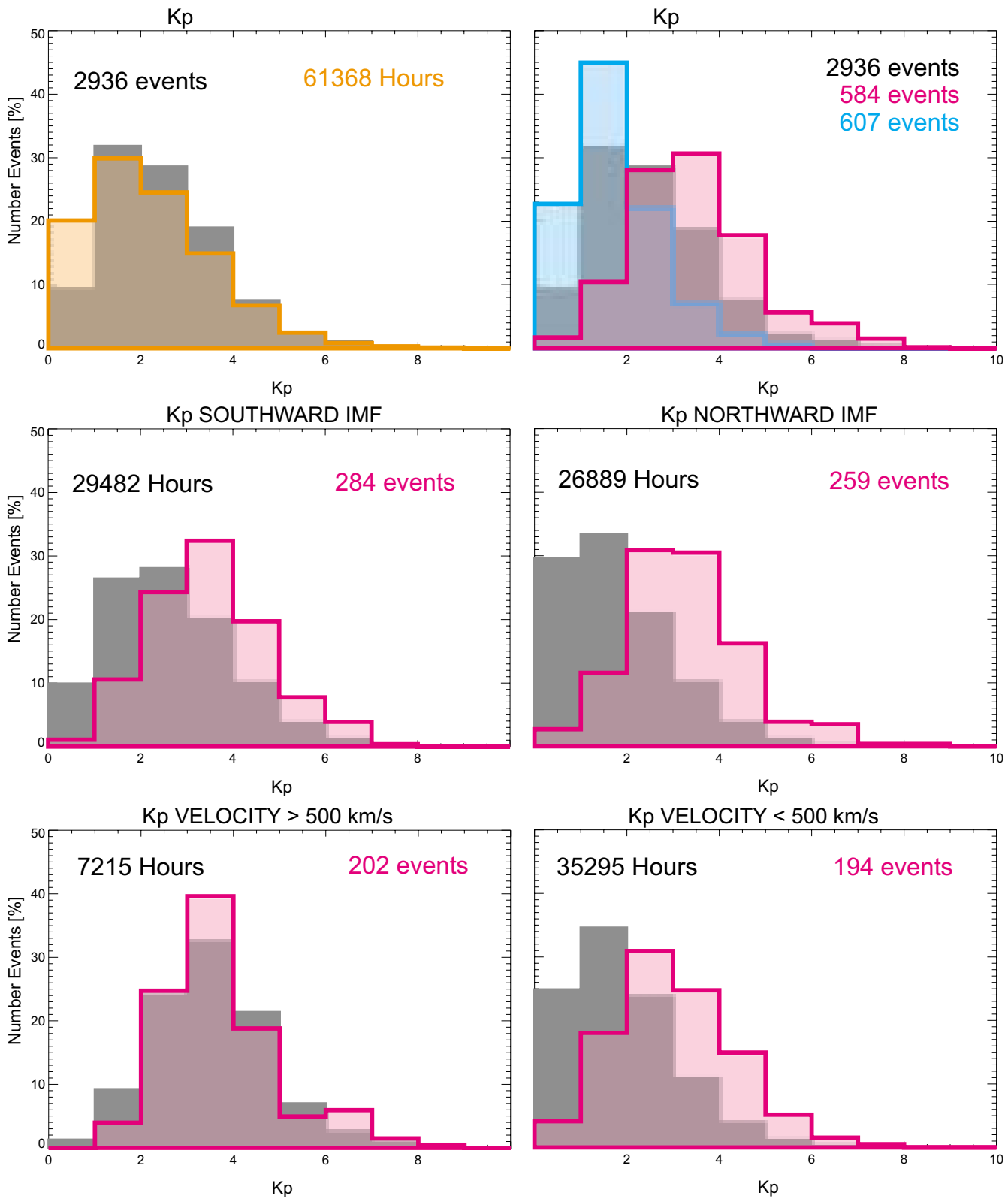

Fig. 14. Relative occurrences as functions of $K_{p}$ at the time of events are displayed. In the top two panels, the gray block diagrams present the distributions for all events. In the top left panel is shown also (orange curve and shading) the background distribution for the entire period covered in the analysis $(61368 \mathrm{~h})$. The panel at the top right displays the separate distributions for the large (magenta) and small (cyan) amplitude subgroups of events. The middle panels display the background distributions (gray block-diagrams) as well as the large amplitude events (magenta curves and shading) for southward (left panel) and northward (right panel) IMF conditions, respectively. Similarly, the bottom panels show the separate distributions for solar wind speeds larger (left panel) and smaller (right panel) than $500 \mathrm{~km} / \mathrm{s}$, respectively.

this that the tendency for events to be associated with higher than average $K_{p}$ values very likely results from the events themselves influencing the $K_{p}$-index value.

\subsection{Average solar wind conditions}

Figure 15 shows the occurrence distributions of events as functions of average solar wind speed and density (top and bottom panels, respectively). In the panels on the left, we show for comparison the corresponding distributions for the entire seven-year interval of the study (orange curves and shading). The panels on the right display the separate distributions for the large (magenta curves and shading) and small (cyan curves and shading) amplitude subgroups of events. Events show a preference for larger than nominal solar wind speeds (top left panel of Fig. 15). We also see that solar wind speed clearly separates the small and large amplitude events (top right panel of Fig. 15). Practically all of the small amplitude events occur for solar wind speeds less than $500 \mathrm{~km} / \mathrm{s}$, whereas more than half of the large ampli- 

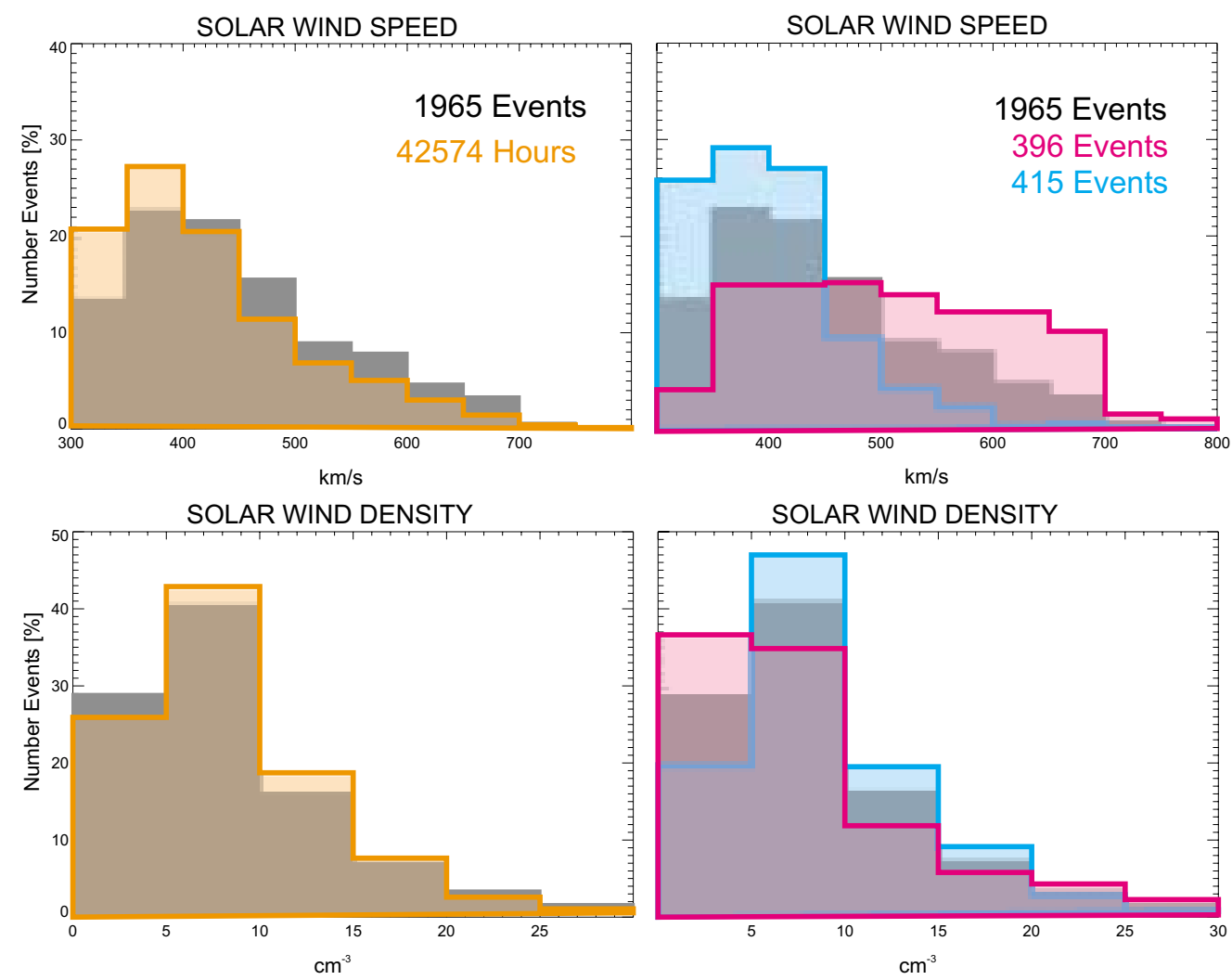

Fig. 15. Relative occurrences as functions of solar wind speed (top panels) and density (bottom panels) at the time of events are displayed. In all panels, the gray block diagrams represent the distributions for all events. For reference, the background distributions for the entire period covered by the analysis ( $42574 \mathrm{~h}$ for which OMNI plasma data exist) are displayed in orange in the panels on the left. Panels on the right display the separate distributions for the large (magenta) and small (cyan) amplitude subgroups of events.

tude events occur for solar wind speeds larger than that. In comparison, the event distribution for the solar wind density (bottom left panel of Fig. 15) is only shifted slightly towards lower densities as compared to the nominal solar wind. The distinction between the small and large amplitude event distributions is also much less pronounced, the large amplitude events occurring slightly more often for lower densities and the small amplitude events for higher densities. This reflects the general tendency for solar wind speed and density to be inversely related (e.g. Hundhausen, 1972).

Sibeck and Korotova (1996) examined hourly averages of the solar wind speed for 152 of their events and concluded that the event distribution did not significantly differ from the background solar wind distribution. Konik et al. (1994) conducted a thorough analysis of the IMF and solar wind plasma conditions for the events of Lanzerotti et al. (1991); they reported a preference for higher than average solar wind speeds for their subset of non-conjugate events (105 events) but not for their conjugate events (56 events). For the solar wind density distributions, the reported results were very close to the ones presented here. We note, however, that the average values for the background solar wind speed and density quoted by Konik et al. (1994) and Lanzerotti et al. (1991) for their study period (July 1985-December 1986) are slightly higher than the ones we find for our seven- year period $(451 \mathrm{~km} / \mathrm{s}$ versus $418 \mathrm{~km} / \mathrm{s}$ for the velocity and $10.7 \mathrm{~cm}^{-3}$ versus $8.9 \mathrm{~cm}^{-3}$ for the density). Large variability is observed in the average solar wind parameters through the solar cycle. Similarly, we observe significant variability in the event distributions for the individual years. This is demonstrated for the solar wind speed in Fig. 16. For each year, 1995 (top panel) to 2000 (bottom panel), the distribution for all events (gray block-diagram), for the large amplitude events (magenta curves and shading) and for the general solar wind for that year (orange curves and shading) are displayed. The event distributions, to a large extent, change in accordance with the average distributions from year to year. For example, all distributions for 1995 are broad and the large amplitude distribution is even double peaked with maximum at high speeds (around $650 \mathrm{~km} / \mathrm{s}$ ). In contrast, all distributions for 1997, including the one for large amplitude events, are much narrower and centered at lower speeds. All years, however, verify the trend of events occurring during higher than average solar wind speeds. Particularly, the large amplitude events seem to have a large variable component associated with high-speed streams in the solar wind. This may also explain the fact that the events occur more frequently during the declining and minimum phases of the solar cycle since this matches the typical behavior of the high-speed solar wind. 


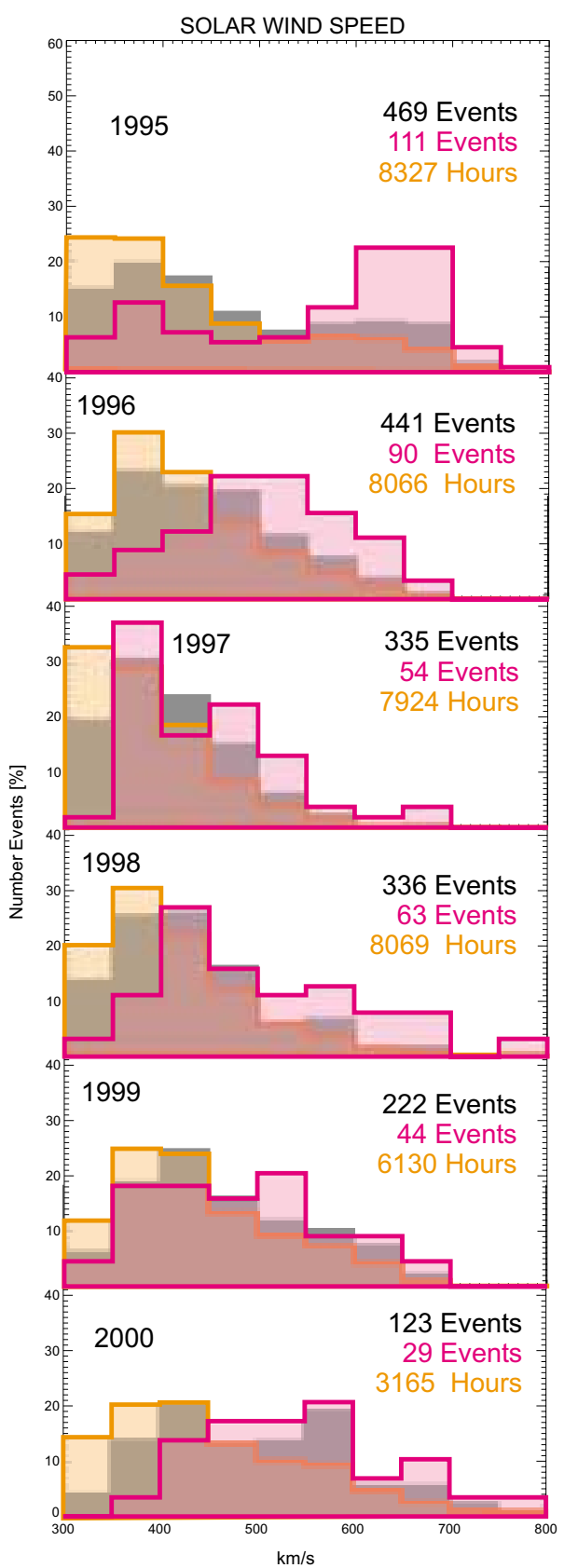

Fig. 16. Relative occurrences as functions of solar wind velocity are displayed for each year individually as marked in the panels. In each panel, the gray block-diagram presents the distribution for all events, while the distribution for the whole year is shown in orange and the separate distribution for the large amplitude events is shown in magenta.

The distributions of the various IMF components for the events as well as for the complete seven year study period are displayed in Figure 17. While the events in general show no specific preference regarding IMF magnitude (top left panel), a distinction is observed between the large and small amplitude subgroups (top right panel) with the large amplitude events occurring for slightly larger than average and the small amplitude for slightly smaller than average IMF magnitudes. All events show slightly enhanced occurrence for small $B_{z}$ (bottom panels) and $B_{y}$ (third row panels) components and for large $B_{x}$ (second row panels) component as compared to the general solar wind. The separate distributions for the large and small amplitude events diverge much less than was observed for the solar wind plasma parameters but do show that for the large amplitude events the general features are further enhanced. The small amplitude distributions, in contrast, show less deviation from the average distributions for the IMF components.

In contrast to the results reported by Konik et al. (1994), Vorobjev et al. (1999), and Sibeck and Korotova (1996) we do not observe any preference for events to occur during northward IMF orientations. Only the small amplitude events occur slightly more frequently for positive than for negative IMF (309 versus 248 events, or about $25 \%$ more). The large amplitude events actually exhibit the opposite trend ( 259 versus 284 events, or roughly $10 \%$ fewer), but this is exactly the same fraction as observed for the IMF for the complete seven-year period. Our results indicate a preference for events to occur for near-radial IMF orientations (large $B_{x}$ and small $B_{y}$ and $B_{z}$ components). A similar result was reported by Konik et al. (1994). In contrast, Sibeck and Korotova (1996) found no preference for near-zero IMF cone angle for their events. We should note, however, that both Konik et al. (1994) and Sibeck and Korotova (1996) reported strong evidence for events to be associated with fluctuations (directional changes) in the IMF, something for which, unfortunately, we have not been able to obtain reliable results from the hourly averages.

\section{Summary and conclusions}

We have presented the results of the first comprehensive statistical study of magnetic impulse events in the high latitude ionosphere using the dense meridional chain of magnetometer stations in Greenland. The identification of events was done purely with an automatic algorithm to avoid the subjectivity introduced by the incorporation of visual inspection of events, on which most previous studies are based (Lanzerotti et al., 1991; Glassmeier et al., 1989; Sibeck and Korotova, 1996; Zesta et al., 2002). The focus is on transient events, requiring variations within a 20-min time-interval to be more than $50 \%$ larger than variations immediately before and after. In addition, requirements are imposed to ensure that the selected events, as far as possible without the use of manual or model-specific verification of vortical structures, are consistent with the magnetic signatures of traveling convection vortices.

Data for the years 1995 through 2001 was processed, yielding 2936 events, of which 584 make up a subgroup of large amplitude ( $>100 \mathrm{nT}$ ) events and 607 a subgroup of small amplitude $(<40 \mathrm{nT})$ events. Considering the differences in station coverage time-period (year) studied and event identification criteria adopted, these numbers are in good agreement with the numbers found in the two most recent comprehensive statistical studies of MIE-events (Sibeck and Korotova, 1996) and TCV-events (Zesta et al., 2002). 

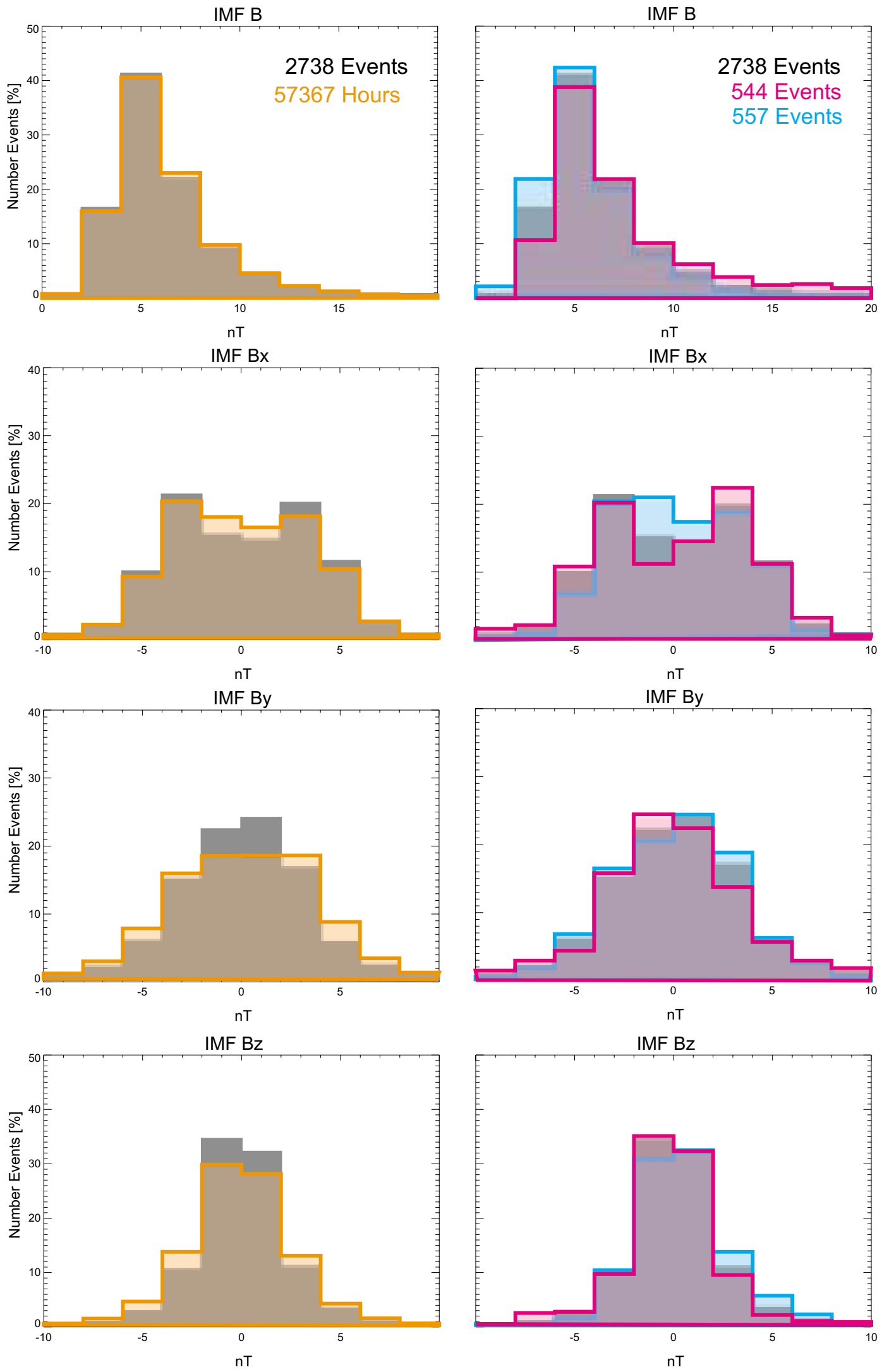

Fig. 17. Relative occurrences as functions of IMF values at the time of events. In all panels, the gray block diagrams present the distributions for all events. For reference, the background distributions for the entire period covered in the analysis $(57367 \mathrm{~h}$ for which OMNI IMF data exist) are displayed in orange for the total field (top) and each component (continuing down) in the panels on the left. In the panels on the right we show the total field (top) and the separate distributions for each component (continuing down) for the large (magenta) and small (cyan) amplitude subgroups of events. 
Examining the occurrence rates of events as a function of month in the year, we find no significant dependence on season. Most prominently, large differences are observed between the distributions for individual years. This may explain the large discrepancies that exist between the earlier studies on this point (Lanzerotti et al., 1991; Glassmeier et al., 1989; Sibeck and Korotova, 1996; Zesta et al., 2002). Furthermore, we find that the latitude of events depends up on dipole tilt angle, such that events occur at higher latitudes for large positive dipole tilt (i.e. during Summer). A study based on a narrow latitude range of stations will, therefore, observe either a reduction in the number of events during summer (for latitudes below roughly $75^{\circ}$ ) or an increase (for latitudes above roughly $75^{\circ}$ ). Our results, for events required to show larger than $30 \mathrm{nT}$ variations at the specific station SKT (at roughly $72^{\circ}$ CGM latitude at the west coast), clearly demonstrate this effect.

Event occurrence, as a function of time of day, peaks broadly around 12:00 UT which corresponds to approximately 10:00 MLT at the west coast of Greenland. Although the incorporation of both east and west coast stations means that we cannot rigorously interpret our results in terms of local time, they are clearly consistent with more events being observed prior to local noon than after. Our results, thus, are largely in agreement with the results of previous studies (Glassmeier et al., 1989; Lanzerotti et al., 1991; Lin et al., 1995; Sibeck et al., 1996; Sibeck and Korotova, 1996; Vorobjev et al., 1999; Zesta et al., 2002). In these, however, the local time distribution of MIE and TCV events has been the subject of intense discussion, mainly concerning the existence, or not, of a mid-day gap in occurrence rates. Our distribution peaks less sharply than those reported earlier and we do not observe a separate secondary peak after noon local time. Two other results of our study shed light on these discrepancies. First, we find a strong relation between event latitude and time of occurrence, such that events are observed at lower latitudes at earlier times. Consequently, as discussed above, if the identification of events is done over a narrow range of latitudes, the resulting local time distribution will depend strongly on the latitude. Latitudes lower than about $75^{\circ}$ will yield a sharp peak in the early morning together with a secondary peak just after noon (examples are the results of Lanzerotti et al., 1991; Sibeck and Korotova, 1996) while higher latitudes will result in a single broader peak later in the morning (examples are the results of Vorobjev et al., 1999; Zesta et al., 2002). Furthermore, we observe that the large amplitude subgroup of events are consistently located at lower than average latitudes and more so at earlier times. As a result, imposing a significant amplitude requirement on the event selection will further sharpen the above latitude effects (an example is the distribution found by Glassmeier et al., 1989). Our results for the SKT subgroup of events demonstrate these effects.

The dependence of latitude on local time clearly resembles that observed for the poleward boundary of the statistical auroral oval or for boundary plasma sheet precipitation at low altitude satellites. This provides strong evidence for the notion that the field-aligned current drivers of the ionospheric MIE events in the ionosphere originate at, and follow as they propagate tailward, a boundary in the outer magnetosphere as suggested by Lühr et al. (1996); Yahnin et al. (1997); Moretto and Yahnin (1998); Moretto et al. (2002). The direction of the dipole tilt-angle effect for the events as well as its overall range are similar to the one observed for the Cusp by Newell and Meng (1989) and larger than what they observe for the LLBL. The asymmetric behavior for positive and negative tilt angles that we observe does not seem consistent with either of their results however. In addition, the local time distribution of events does not make the Cusp a likely candidate for the source region.

Our events tend to occur at slightly elevated $K_{p}$ values. The overall average for events is $2^{+}$as compared to $2^{0}$ for the general distribution. This is in good agreement with results quoted in earlier studies (Glassmeier et al., 1989; Lanzerotti et al., 1991; Konik et al., 1994; Zesta et al., 2002). Geomagnetic activity level clearly separates the small and large amplitude subgroups. The average is $2^{-}$for small amplitude events and $3^{+}$for large amplitude events. However, we also find that high $K_{p}$ values are about equally likely for large amplitude events during northward as during southward IMF and that more high $K_{p}$ values are associated with the lower than with the higher solar wind speed. We conclude from this that the tendency for events to be associated with higher than average $K_{p}$ values is most likely a result of the events themselves contributing to the $K_{p}$-index value. This can be confirmed by analyzing the direct response to high-latitude MIE events in stations at lower latitudes and will be the subject of a future study.

From year to year, the distributions of events versus solar wind speed resemble the distributions for solar wind speed themselves. Nevertheless, all years, exhibit a clear trend for events to occur during higher than average solar wind speeds. In particular, a large but variable fraction of the high amplitude events seems to be associated with high-speed streams in the solar wind. Consequently, they exhibit increased occurrence during the declining and minimum phases of the solar cycle typical for the variation of the high-speed solar wind.

In contrast to the results reported by Konik et al. (1994), Vorobjev et al. (1999), and Sibeck and Korotova (1996) we do not observe a preference for events to occur during northward IMF orientations. Events tend to occur for near-radial IMF orientations (large $B_{x}$, but small $B_{y}$ and $B_{z}$ components) similar to the result reported by Konik et al. (1994). In contrast, Sibeck and Korotova (1996) found no preference for near-zero IMF cone angle for their events. We note, however, that both Konik et al. (1994) and Sibeck and Korotova (1996) reported strong evidence for events to be associated with fluctuations (directional changes) in the IMF, something for which, unfortunately, we have not been able to obtain reliable results based on the hourly averages

Evidence from several case studies (Sitar et al., 1998; Sibeck et al., 1999; Moretto et al., 2002; Murr et al., 2002) together with the statistical study of Murr and Hughes 
(2003) seem to confirm the possibility that dynamic pressure changes associated with cavities in the foreshock (created by back-streaming ions reflected at the bow shock) could be the main source of MIEs (e.g. Sibeck, 1995). Our results here are consistent with this in several ways. TCVs occur more frequently prior to local noon than after. The same was found for the observation of foreshock cavities by Sibeck et al. (2001), who explain this as a result of the IMF spiral orientation providing longer-lasting magnetic connections to the bowshock in the pre-noon sector. They also report that foreshock cavities exhibit a preference for high-speed solar wind streams and radial IMF orientation in a manner very similar to the preferences reported here for our MIE events.

Statistical results concerning the detailed characteristics of the TCV events, such as their vortical structure and propagation properties, would help to further distinguish between the various proposed generation mechanisms. The determination of the detailed features of the events, however, requires individual (manual) analysis of each event and hence has not been pursued for this study. Rather, we propose this as the subject of a future study based on a subset of the events, for which high-resolution solar wind measurements are also available.

Acknowledgements. The magnetometer array in Greenland was constructed and is operated by the Danish Meteorological Institute. Observations of the average near-Earth solar wind magnetic field and plasma conditions for this study was obtained through the OMNI database facility provided by the National Space Science Data Center at NASA GSFC. We gratefully acknowledge this service as well as the satellite missions that contribute to it. T. Moretto was supported by NSF grant ATM-0096534 to the John Hopkins University, Applied Physics Laboratory. We thank A. T. Lui there for his support. D. G. Sibeck was supported by NASA grant NAG510479.

Topical Editor T. Pulkkinen thanks R. Clauer and A. Yahnin for their help in evaluating this paper.

\section{References}

Amm, O., Engebretson, M. J., Hughes, T., Newitt, L., Viljanen, A., and Watermann, J.: A traveling convection vortex event study: Instantaneous ionospheric equivalent currents, estimation of field-aligned currents, and the role of induced currents, J. Geophys. Res., 107, 1334, doi:10.10292002JA009472, 2002.

Clauer, C. R. and Petrov, V. G.: A statistical investigation of traveling convection vortices observed by the west coast Greenland magnetometer chain, J. Geophys. Res., 107, 10.10292001JA000 228, 2002.

Feldstein, Y. I.: On morphology of auroral and magnetic disturbances at high latitudes, Geomagn. Aeron., 3, 183, 1963.

Friis-Christensen, E., McHenry, M. A., Clauer, C. R., and Vennerstrøm, S.: Ionospheric traveling convection vortices observed near the polar cleft: A triggered response to sudden changes in the solar wind, Geophys. Res. Lett., 15, 253-256, 1988.

Glassmeier, K.-H., Lester, M., Mier-Jedrzejowicz, W. A. C., Green, C. A., Rostoker, G., Orr, D., Wedeken, U., Junginger, H., and Amata, E.: Pc5 pulsations and their possible source mechanisms: A case study, J. Geophys., 55, 108-119, 1984.
Glassmeier, K.-H., Hönisch, M., and Untiedt, J.: Ground-based and satellite observations of traveling magnetospheric convection twin vortices, J. Geophys. Res., 94, 2520-2528, 1989.

Gustafsson, G., Papitashvili, N. E., and Papitashvili, V. O.: A revised corrected geomagnetic coordinate system for Epochs 1985 and 1990, J. Atmos. Terr. Phys., 54, 1609-1631, 1992.

Holzworth, R. H. and Meng, C.-I.: Mathematical representation of the auroral oval, Geophys. Res. Lett., 2, 377, 1975.

Hundhausen, A. J.: Coronal expansion and solar wind, SpringerVerlag, New York, 1972.

Kataoka, R., Fukunishi, H., Lanzerotti, L. J., Maclennan, C. G., Frey, H. U., Mende, S. B., Doolittle, J. H., Rosenberg, T. J., and Weatherwax, A. T.: Magnetic impulse events: A detailed case study of extended ground and space observations, J. Geophys. Res., 106, 25 873, 2001.

Konik, R. M., Lanzerotti, L. J., Wolfe, A., Maclennan, C. G., and Venkatesan, D.: Cusp latitude magnetic impulse events, 2, Interplanetary magnetic field and solar wind conditions, J. Geophys. Res., 99, 14 831-14 853, 1994.

Lanzerotti, L. J., Maclennan, C. G., Medford, L. V., Lee, L. C., and Wolfe, A.: Possible evidence of flux transfer events in the polar ionosphere, Geophys. Res. Lett., 13, 1089-1092, 1986.

Lanzerotti, L. J., Konik, R. M., Wolfe, A., Venkatesan, D., and MacLennan, C. G.: Cusp latitude magnetic impulsive events, 1, Occurrence statistics, J. Geophys. Res., 96, 14 009-14 022, 1991.

Lin, Z. M. et al.: Statistical studies of impulsive events at high latitudes, J. Geophys. Res., 100, 7553-7566, 1995.

Lühr, H. and Blawert, W.: Ground signatures of travelling convection vortices, in Solar Wind Sources of Magnetospheric ULF Waves,Geophys. Monogr. Ser., edited by M. J. Engebretson, K. Takahashi, and M. Scholer, vol. 81, pp. 231-251, AGU, Washington, D. C., 1994.

Lühr, H., Lockwood, M., Sandholt, P. E., Hansen, T. L., and Moretto, T.: Multi-instrument ground-based observations of a travelling convection vortices event, Ann. Geophys., 14, 162$181,1996$.

Lühr, H., Rother, M., Iyemori, T., Hansen, T. L., and Lepping, R. P.: Superposed epoch analysis applied to large-amplitude travelling convection vortices, Ann. Geophys., 16, 743-753, 1998.

Moretto, T. and Yahnin, A.: Mapping Travelling Convection Vortex Events With Respect to Energetic Particle Boundaries, Ann. Geophys., 16, 891-899, 1998.

Moretto, T., Friis-Christensen, E., Lühr, H., and Zesta, E.: Global Perspective of Ionospheric Travelling Convection Vortices: Case Studies of Two GEM Events, J. Geophys. Res., 102, 11597 $11610,1997$.

Moretto, T., Hesse, M., Yahnin, A., Ieda, A., Murr, D., and Watermann, J. F.: Magnetospheric signature of an ionospheric traveling convection vortex event, J. Geophys. Res., 107, 10.10292001JA000 049, 2002.

Murr, D., Hughes, W. J., Rodger, A. S., Zesta, E., Frey, H. U., and Weatherwax, A. T.: Conjugate observations of traveling convection vortices: The field-aligned current system, J. Geophys. Res., 107, 10.10292002JA009 456, 2002.

Murr, D. L. and Hughes, W. J.: Solar wind drivers of Traveling Convection Vortices, Geophys. Res. Lett., 30, 1354, doi:10.1029/2002GL015498, 2003.

Newell, P. T. and Meng, C.-I.: Dipole tilt angle effects on the latitude of the cusp and cleft/low-latitude boundary layer, J. Geophys. Res., 94, 6949, 1989.

Newell, P. T. and Meng, C.-I.: Mapping the dayside ionosphere to the magnetosphere according to particle precipitation 
characteristics, Geophys. Res. Lett., 19, 609, 1992.

Papitashvili, N. E. and King, J. H.: Corrected geomagnetic software available, Tech. Rep. NSSDC News 9, NSSDC at NASA/Goddard Space Flight Center, the programs can either be run interactively from NSSDC by choosing the models option of the main NODIS menu (telnet nssdca.gsfc.nasa.gov: username $=$ nodis ) or the Fortran code can be retrieved via Anonymous FTP to nssdca.gsfc.nasa.gov, Username $=$ anonymous, Password $=$ remotenetworkaddress. Cd to models/geo_cgm., 1993.

Sibeck, D. G.: Solar wind dynamic pressure variations: Quantifying the statistical magnetospheric response, in Plasma Astrophysics,Eur. Space Agency Spec. Publ., edited by T. D. Guyenne and J. J. Hunt, vol. SP-311, 63-68, 1990.

Sibeck, D. G.: Transient Magnetic Field Signatures at High Latitudes, J. Geophys. Res., 98, 243-256, 1993.

Sibeck, D. G.: The magnetospheric response to foreshock pressure pulses, in Physics of the Magnetopause,Geophys. Monogr. Ser., edited by P. Song, B. U. Ö. Sonnerup, and M. F. Thomsen, vol. 90, pp. 293-302, AGU, Washington, D. C., 1995.

Sibeck, D. G. and Korotova, G. I.: Occurrence patterns for transient magnetic field signatures at high latitudes, J. Geophys. Res., 101, 13 413-13 428, 1996.

Sibeck, D. G., Baumjohann, W., and Lopez, R. E.: Solar wind dynamic pressure variations and transient magnetospheric signatures, Geophys. Res. Lett., 16, 13-16, 1989.

Sibeck, D. G., Greenwald, R. A., Bristow, W. A., and Korotova, G. I.: Concerning Possible Effects of Ionospheric Conductivity Upon the Occurrence Patterns of Impulsive Events in HighLatitude Ground Magnetograms, J. Geophys. Res., 101, 13 407$13412,1996$.

Sibeck, D. G. Borodkova, N. L., Schwartz, S. J., Owen, C. J., Kessel, R., Kokubun, S., Lepping, R. P., Lin, R., Liou, K., Lúhr, H., McEntire, R. W., Meng, C.-I., Mukai, T., Nemecek, Z., Parks, G., Phan, T. D., Romanov, S. A., Safrankova, J., Sauvaud, J.-A., Singer, H. J., Solovyev, S. I., Szabo, A., Takahashi, K., Williams,
D. J., Yumoto, K., Zastenker, G. N. : Comprehensive study of the magnetospheric response to a hot flow anomaly, J. Geophys. Res., 104, 4577-4593, 1999.

Sibeck, D. G., Decker, R. B., Mitchell, D. G., Lazarus, A. J., Lepping, R. P., and Szabo, A.: Solar wind preconditioning in the flank foreshock: IMP8 observations, J. Geophys. Res., 106, 21 675-21 688, 2001.

Sitar, R. J., Baker, J. B., Clauer, C. R., Ridley, A. J., Cumnock, J. A., Papitashvili, V. O., Spann, J., Britnacher, M. J., and Parks, G. K.: Multi-instrument analysis of the ionospheric signatures of a hot flow anomaly occurring on July 24, 1996, J. Geophys. Res., 103, 23 357-23 372, 1998.

Southwood, D. J. and Kivelson, M. G.: Magnetospheric interchange motions, J. Geophys. Res., 94, 299, 1989.

Southwood, D. J. and Kivelson, M. G.: An approximate description of field-aligned currents in a planetary magnetic field, J. Geophys. Res., 96, 67, 1991.

Vorobjev, V. G., Yagodkina, O. I., and Zverev, V. L.: Morphological features of bipolar magnetic impulsive events and associated interplanetary medium signatures, J. Geophys. Res., 104, 45954607, 1999.

Wilhjelm, J. and Friis-Christensen, E.: Magnetometer chain in Greenland, Geoph. Pap. R-48, Danish Meteorological Institute, Copenhagen, Denmark, 1976.

Yahnin, A. G., Vorobjev, V. G., Bösinger, T., Rasinkangas, R., Sibeck, D. G., and Newell, P. T.: On the source region of the travelling convection vortices, Geophys. Res. Lett., 24, 237-240, 1997.

Zesta, E., Hughes, W. J., Engebretson, M. J., Hughes, T. J., Lazarus, A. J., and Paularena, K. I.: The November 9, 1993 traveling convection vortex event: A case study, J. Geophys. Res., 104, $28041,1999$.

Zesta, E., Hughes, W. J., and Engebretson, M. J.: A statistical study of traveling convection vortices using the Magnetometer Array for Cusp and Cleft Studies, J. Geophys. Res., 107, 1317, doi:10.10291999JA000386, 2002. 